\title{
SUPERFÍCIES DE TIPO MÍNIMO EM $S^{3}$
}

\author{
Roseli Nozaki Grave de Andrade
}

TESE DEFENDIDA

NO

INSTITUTO DE MATEMÁTICA E ESTATÍSTICA

DA

UNIVERSIDADE DE SÀO PAULO

PARA

OBTENÇÃO DO GRAU DE DOUTOR

EM

MATEMÁTICA

Área de Concentraçào: Geometria

Orientador: Prof. Dr. Fabiano Gustavo Braga Brito

Sào Paulo, julho de 1994 - 


\title{
SUPERFÍCIES DE TIPO MÍNIMO EM $\varsigma^{3}$
}

\author{
Este exemplar corresponde à redaçào \\ da tese devidamente corrigida \\ e defendida por Roseli Nozaki Grave de Andrade \\ e aprovada pela Comissào Julgadora.
}

São Paulo, 09 de janeiro de 1995.

Banca Examinadora:

Prof. Dr. Fabiano Gustavo Braga Brito (orientador)

Prof. Dr. Plínio Amarante Quirino Simões

Prof. Dr. Jaime Bruck Ripoll

Prof. Dr. Ricardo Sá Earp

Prof. Dr. Francesco Mercuri 


\section{Resumo}

Introduzimos a noçào de superfície de Weingarten de tipo mínimo em $\varsigma^{3}$. Aplicando algumas técnicas da Geometria Equivariante, construímos exemplos rotacionais desses objetos e estudamos o "problema do mergulho"

\section{Abstract}

We introduce the notion of minimal type Weingarten surface in $S^{3}$. Applying some Equivariant Geometry techniques we construct rotational examples of those objects and we study the embeddness problem. 
Aos meus queridos

Paulo, Luciana e Tatiana 


\section{Agradecimentos}

Ao amigo e orientador Fabiano Brito, pela oportunidade, pelas diretrizes seguras, pelo permanente incentivo e pelas valiosas conversas matemáticas.

À minha màe: uma fonte constante de amor, segurança, coragem e força.

Aos amigos, pelos momentos de alegre descontraçào e pelo auxílio e encorajamento nas horas de dificuldade.

Ao DMA-CCE-UEM, pela oportunidade e sem o apoio do qual este trabalho nào se realizaria.

Aos colegas Luiz Henry e Edson por algumas estimativas numéricas.

Ao Walter, pelo excelente trabalho de edição.

A todos que direta ou indiretamente colaboraram na execução deste trabalho. 


\section{Índice}

Introdução

Capítulo 1

Preliminares

1.1 Classes de superfícies especiais invariantes pela aplicaçào de Gauss em $S^{3} \ldots 5$

1.2 Sobre a EDO que determina a curva geradora de uma superfície de

Weingarten especial rotacional em $S^{3}$

\section{Capítulo 2}

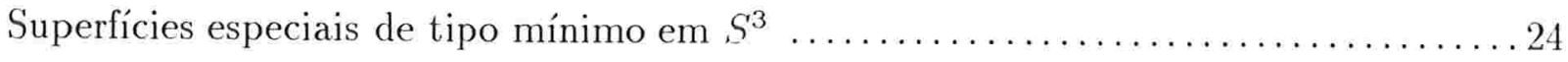

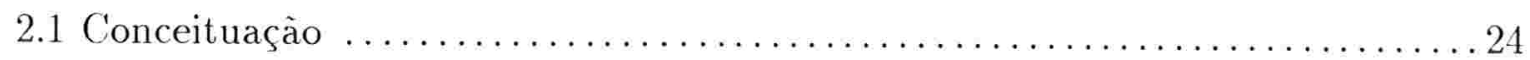

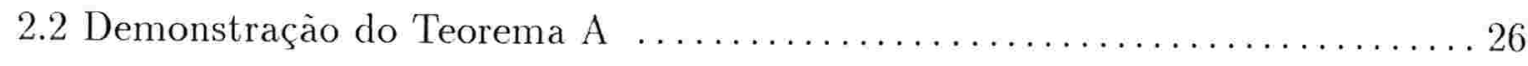

\section{Capítulo 3}

Sobre certas superfícies rotacionais especiais de tipo mínimo em $S^{3} \ldots \ldots \ldots \ldots . \ldots 37$

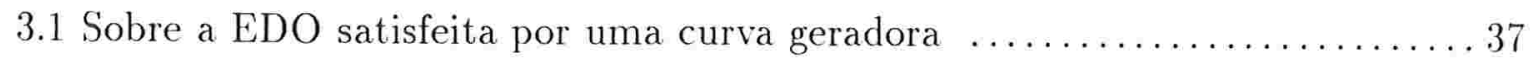

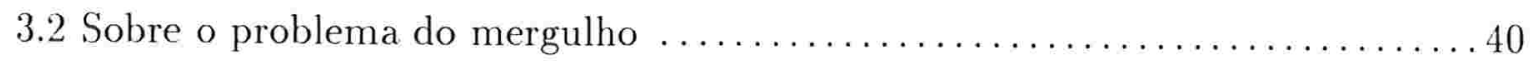




\section{Introdução}

Existe algum toro minimo mergulhado $\mathrm{em} \mathrm{S}^{3}$ além do toro de Clifford?

A resposta a esta pergunta é desconhecida. Mergulhos mínimos de superfícies compactas de maior gênero em $S^{3}$ foram introduzidos por $B$. Lawson [L]. Além disso, pelo teorema de Hopf [Ho], as esferas totalmente geodésicas sào os únicos exemplos de imersòes mínimas de superfícies de gênero $0 \mathrm{em} S^{3}$.

As superfícies rotacionais sào, num certo sentido, aquelas que representam a classe natural de exemplos de superfícies mínimas em $S^{3}$, uma vez estudadas as homogêneas. A busca de um contra-exemplo para a pergunta inicial deveria pois começar pelas rotacionais. Isso foi feito em [O1], [HL], [M] e [R2]. Verificou-se que as únicas superfícies compactas mínimas rotacionais mergulhadas em $S^{3}$ são as 2-esferas totalmente geodésicas e o toro de Clifford. Mergulhos rotacionais de superfícies compactas de curvatura média constante $H$ em $S^{3}$ podem ser vistos, por exemplo, em [R1], para todo valor positivo de $H \neq \frac{\sqrt{3}}{3}$.

Uma propriedade curiosa das superfícies mínimas em $S^{3}$ é a da invariância pela aplicaçào de Gauss. Precisamente, seja $M^{2} \hookrightarrow S^{3}$ uma imersào mínima e $g: M^{2} \rightarrow S^{3}$ a aplicação normal de Gauss que associa a cada ponto $p \in M^{2}$ o vetor normal unitário $\mathbf{n}(p)$, tangente a $S^{3}$ e normal a $M^{2}$ no ponto $p$. Se a segunda forma fundamental, $I I(p)$, de $M^{2}$ em $p$ é não degenerada, digamos, $\operatorname{det}\left(A_{\mathbf{n}}(p)\right) \neq 0\left(A_{\mathbf{n}}(p)\right.$ é a aplicaçào linear auto-adjunta associada a $I I(p))$, então $g\left(M^{2}\right)$ é uma superfície regular de $S^{3}$. Nessas condições, se $M$ é mínima, entào $g(M)$ também é mínima. Tal invariância não se dá, por exemplo, na classe das superfícies de curvatura média constante.

No primeiro capítulo deste trabalho introduzimos as classes de superfícies especiais de Weingarten que satisfazem essa invariância. 
Chamamos superfície especial de Weingarten a uma superfície $M \hookrightarrow S^{3}$ cujas curvaturas, média e de Gauss-Kronecker, denotadas respectivamente por $H$ e $K_{\epsilon}$. satisfazem uma relaçào do tipo

$$
H=f\left(H^{2}-K_{\epsilon}\right) \text {. }
$$

onde $f:[0,+\infty[\rightarrow \mathbb{R}$ é uma funçào contínua em $[0,+\infty[$ e real analítica em $] 0,+\infty[$.

Superfícies especiais de Weingarten foram estudadas por P.Hartman \& A. Wintner ([HW]), S. S. Chern ([C]), H. Hopf ([Ho]) e R. Bryant ([B]). Recentemente, em [SaET1], R. Sá Earp e E. Toubiana introduziram a noçào de superfície especial de Weingarten de tipo mínimo em $\mathbb{R}^{3}$. Basicamente, uma tal classe de superfícies satisfaz o Princípio do Máximo e contém os planos de $\mathbb{R}^{3}$. Tal conceito estende-se a $\mathbb{H}^{3}$ sem modificaçòes básicas, porém, com resultados surpreendentes.

Introduzimos, no Capítulo 2, a noção de superfícies de tipo mínimo em $S^{3}$.

As classes de superfícies de Weingarten especiais em $S^{3}$ invariantes pela aplicaçào de Gauss, que obtemos na Proposição 1.1.2 do Capítulo 1, serão denominadas classes de superfícies de Weingarten de tipo mínimo, tendo em vista as seguintes analogias com a classe das superfícies mínimas (além daquela dada por tal invariância):

1. $f(0)=0$, ou seja, as 2-esferas totalmente geodésicas estão na classe.

2. Sobre cada superfície fechada $M$, em qualquer das classes obtidas, a curvatura $K_{\epsilon}$ de Gauss-Kronecker é negativa.

3. Em cada tal classe existe um único toro flat mergulhado em $S^{3}$.

Utilizamos a técnica da Geometria Equivariante para estudar qualitativamente superfícies rotacionais especiais de tipo mínimo em $S^{3}$, uma vez que estas são invariantes pela ação de um certo subgrupo $G$ das isometrias de $S^{3}$. Desta forma, o estudo de uma tal 
superfície se reduz à análise de uma certa equaçào diferencial de segunda ordem satisfeita pela curva geradora de uma tal superfície, no espaço de órbitas, $S^{3} / G$. da açào de $G$ sobre $S^{3}$.

O principal resultado que obtemos no Capítulo 2 é o seguinte:

Teorema A Em cada classe de superfícies especiais de tipo mínimo, existe uma quantidade infinita de superfícies compactas de gênero um, imersas em $S^{3}$.

O Capítulo 3 é reservado ao estudo das classes de superfícies especiais rotacionais dadas por funçôes $f$ do tipo $f(x)=c \sqrt{x}$, onde $c$ é uma constante real no intervalo $] 0,1[$. A importância do estudo particular de tais classes de superfícies é a obtençào do seguinte teorema:

Teorema B Em cada classe de superfícies especiais de tipo mínimo, dada por uma função do tipo $f(x)=c \sqrt{x}, c \in] 0,1[$, a única superfície especial compacta de gênero um e mergulhada em $S^{3}$ é o toro flat

$$
S^{1}\left(\sqrt{\frac{a}{\alpha+1}}\right) \times S^{1}\left(\sqrt{\frac{1}{\alpha+1}}\right),
$$

onde $\alpha=\frac{1+c}{1-c}$.

O ponto crucial da demonstração do teorema B é a apresentação de estimativas para os períodos de curvas geradoras de tais superfícies rotacionais de tipo mínimo em $S^{3}$. Curiosamente as equaçòes diferenciais de segunda ordem correspondentes às superfícies de tipo mínimo das classes $f(x)=c \sqrt{x}, 0<c<1$, sào semelhantes às das hipersuperfícies mínimas de Otsuki em $S^{n+1}$ (veja [O1]). Utilizamos a técnica desenvolvida por Otsuki e Furuya em [O1], [O2] e [F], para estimar os períodos das curvas geradoras das superfícies em questão.

Fixada uma classe de superfícies de tipo mínimo em $S^{3}$, o problema da existência, ou 
nào, de exemplos rotacionais compactos mergulhados, além do único toro flat mergulhado em $S^{3}$, permanece ainda sem resposta. O que apresentamos no Capítulo 3 é uma resposta de existência desses exemplos para classes particulares de superfícies de tipo mínimo. classes estas dadas por inroluçòes cujos gráficos sào certas hipérboles. Numa tal classe vale a condiçào de elipticidade para a equaçào diferencial parcial correspondente. 


\section{Capítulo 1}

\section{Preliminares}

\subsection{Classes de superfícies especiais invariantes pela aplicação de Gauss em $S^{3}$}

Seja $S^{3} \subset \mathbb{R}^{4}$ a esfera euclideana unitária.

Seja $M^{2} \hookrightarrow S^{3}$ uma superfície conexa, orientável, imersa em $S^{3}$.

Para cada $p \in M$, o produto interno $\langle,\rangle_{p}$, em $T_{p} S^{3}$ o decompòe na soma direta $T_{p} S^{3}=T_{p} M \oplus\left(T_{p} M\right)^{\perp}$, onde $\left(T_{p} M\right)^{\perp}$ denota o complemento ortogonal de $T_{p} M$ em $T_{p} S^{3}$. Segundo esta decomposiçào, se $v \in T_{p} S^{3}$, escrevemos $v=v^{\top}+v^{\perp}$, onde $v^{\top}$ e $v^{\perp}$ sào vetores, respectivamente, em $T_{p} M$ e $\left(T_{p} M\right)^{\perp}$. Escolhamos $\mathbf{n}(p)$ em $\left(T_{p} M\right)^{\perp},|\mathbf{n}(p)|=1$, de modo que se $\left\{v_{1}, v_{2}\right\}$ é uma base na orientação de $M$ então $\left\{v_{1}, v_{2}, \mathbf{n}\right\}$ é uma base na orientação de $S^{3}$, esta última sendo dada pelo campo normal unitário $N(x)=-x$, $\left(x \in S^{3}\right)$.

Definamos uma aplicação $g: M \rightarrow S^{3}$ como segue. O vetor unitário normal $\mathbf{n}(p)$, na origem de $\mathbb{R}^{4}$, determina um ponto. também denotado por $\mathbf{n}(p)$, em $S^{3}$. Definimos a aplicaçào $g$ por $g(p)=\mathbf{n}(p) \in S^{3}$. Geometricamente, $g(p)$ é o ponto sobre a geodésica 
de $S^{3}$ passando por $p$ e ortogonal a $M$. na direçào de $\mathbf{n}(p)$, obtido a partir de $p$ por uma rotaçào de $\frac{\pi}{2}$.

A diferencial. $\mathrm{d} g_{p}$. de $g$ em $p$, pode ser vista como uma aplicaçào de $T_{p} M \mathrm{em} T_{p} M$. De fato, $\mathrm{d} g_{p}$, geometricamente, transporta $T_{p} M$ ao longo de uma geodésica de $S^{3}$. ortogonal a $M$, na direção de $\mathbf{n}(p)$. segundo um ângulo de $\frac{\pi}{2}$, sobre um subespaço de $T_{g(p)}, S^{3}$ paralelo a $T_{p} M$. E mais, se $\overline{\mathbf{n}}$ é uma extensào local de $\mathbf{n}$ a $S^{3}$, unitária e normal a $M$, entào

$$
\mathrm{d} g_{p}(v)=\frac{\mathrm{d}}{\mathrm{d} t}(g \circ c(t))_{t=0}=\frac{\mathrm{d}}{\mathrm{d} t}(\overline{\mathbf{n}} \circ c(t))_{t=0}=\bar{\nabla}_{v} \overline{\mathbf{n}},
$$

onde $\bar{\nabla}$ é a conexào Riemanniana de $S^{3}, c:(-\epsilon, \epsilon) \rightarrow M$ é uma curva $\operatorname{com} c(0)=p$ e $c^{\prime}(0)=v$. Usando que $\langle\overline{\mathbf{n}}, \overline{\mathbf{n}}\rangle=1$ temos que $\bar{\nabla}_{v}, \overline{\mathbf{n}}=\left(\bar{\nabla}_{v}, \overline{\mathbf{n}}\right)^{\top}$, de modo que $\mathrm{d} g_{p}(v)=$ $\left(\bar{\nabla}_{v} \overline{\mathbf{n}}\right)^{\top}=-A_{\mathbf{n}}(v)$, onde $A_{\mathrm{n}}$ é a aplicaçào linear auto-adjunta associada à segunda forma fundamental $I_{\mathbf{n}}$ de $M$. Portanto, para a aplicaçào $g$ valem propriedades como as de uma aplicaçào normal de Gauss de uma superfície em $\mathbb{R}^{3}$.

Para cada $p \in M$, existe uma base ortonormal $\left\{\epsilon_{1}, \epsilon_{2}\right\}$ de $T_{p} M$ de autovetores com autovalores $\lambda_{1}, \lambda_{2}$ de $A_{1}$, em $p$, os quais são, respectivamente, as direçòes principais e as curvaturas principais de $M$ em $p$. Temos entào que $\mathrm{d} g_{p}\left(e_{i}\right)=-\lambda_{i} e_{i}, i=1,2$. As funções simétricas $K_{\epsilon}\left(\lambda_{1}, \lambda_{2}\right)$ e $H\left(\lambda_{1}, \lambda_{2}\right)$, denominadas respectivamente curvatura de Gauss-Kronecker e curvatura média de $M$ em $p$, são dadas por

$$
K_{\epsilon}(p)=\operatorname{det}\left(\mathrm{d} g_{p}\right) \quad \text { e } H(p)=-\frac{1}{2} \operatorname{traço}\left(\mathrm{d} g_{p}\right) .
$$

Observemos que se $K_{\epsilon}(p)=\operatorname{det}\left(\mathrm{d} g_{p}\right) \neq 0$, para todo $p$ em $M$, entào $g$ é um difeomorfismo local e, portanto, a imagem $M^{*}$ de $M$ por $g$ é uma superfície em $S^{3}$. Orientamos $M^{*}$ como segue. Se $p \in M \subset S^{3}$, definimos a aplicação $g$ fazendo $g(p)=\mathbf{n}(p)$, onde $\mathbf{n}(p)$ significa aqui o vetor posiçào do ponto $g(p)$ em $S^{3}$, ou seja, $\mathbf{n}(p)$ é normal a $T_{g(p)} S^{3}$. Por outro lado, $\mathbf{n}(p)$ é um vetor de $T_{p} S^{3}$ e assim. $\langle\mathbf{n}(p), p\rangle=0$, onde $p$ agora significa o vetor posiçào do ponto $p \mathrm{em} S^{3}$. Então, se v $\in T_{p} M$ temos que $\left\langle\bar{\nabla}_{v} \mathbf{n}, p\right\rangle_{T_{p} S^{3}}=0$ e, portanto, que $\left\langle\mathrm{d} g_{p}(v), p\right\rangle_{T_{g(p)} S^{3}}=0$. Desta forma. $p$ (bem como $-p$ ) é normal a $M^{*}$ em $g(p)$. Façamos a 
escolha para o vetor normal a $M^{*}$ em $g(p)$ pondo $\mathbf{n}^{*}(g(p))=p$ ou $-p .+$ ou - dependendo de ser o sinal de det $\left(\mathrm{d} g_{p}\right)$ respectivamente positivo ou negativo. Tal escolha é baseada no fato de que se $\left\{v_{1}, v_{2}\right\}$ é uma base na orientaçào de $M$. entào $\left\{\mathrm{d} g_{p} v_{1}, \mathrm{~d} g_{p} v_{2}, \mathrm{n}^{*}(g(p))\right\}$ deve ser uma base na orientaçào de $S^{3}$ em $g(p)$.

Sendo $M$ conexa, o sinal de $K_{\epsilon}(p)=\operatorname{det}\left(\mathrm{d} g_{p}\right)$ é sempre positivo ou sempre negativo, de modo que a aplicaçào de Gauss, $g^{*}: M^{*} \rightarrow S^{3}$. de $M^{*}$ em $S^{3}$, pode ser dada por

$$
g^{*}\left(p^{*}\right)= \begin{cases}p & , \text { para todo } p^{*}=g(p) \text { em } M^{*} \text { e } K_{\epsilon}(p)>0 \\ -p & , \text { para todo } p^{*}=g(p) \text { em } M^{*} \text { e } K_{\epsilon}(p)<0 .\end{cases}
$$

A diferencial, $\mathrm{d} g_{p^{*}}^{*}$, pode ser vista novamente como uma aplicaçào $T_{p^{*}} M^{*} \rightarrow T_{p^{*}} M^{*}$ que, pelo paralelismo de $T_{p^{*}} M^{*}$ e de $T_{p} M$, pode ser, por sua vez, vista como uma aplicaçào $T_{p} M \rightarrow T_{p} M$. Desta forma, temos que $\mathrm{d} g_{p^{*}}^{*}=\left(\mathrm{d} g_{p}\right)^{-1}$ ou $-\left(\mathrm{d} g_{p}\right)^{-1}$, onde + ou depende respectivamente de ser $K_{\epsilon}(p)>0$ ou $K_{\epsilon}(p)<0$.

Podemos resumir todas estas consideraçòes na seguinte proposiçào.

Proposição 1.1.1 Seja $M \hookrightarrow S^{3}$ uma superfície conexa orientável. Se a aplicaçâo de Gauss $g$ em $S^{3}$ é tal que $\operatorname{det}\left(\mathrm{d} g_{p}\right) \neq 0$, para todo $p$ em $M$, entâo $M^{*}=g(M)$ é uma superficie. E mais, se $\mathbf{n}$ e $\mathbf{n}^{*}$ indicam os respectivos vetores normais de $M$ em $p$ e de $M^{*}$ em $p^{*}=g(p)$, entâo

$$
A_{\mathrm{n}^{*}}^{*}=A_{\mathrm{n}}^{-1} \quad \text { ou } \quad-A_{\mathrm{n}}^{-1}
$$

$\left(+\right.$ ou - dependendo de $\operatorname{det}\left(\mathrm{d} g_{p}\right)>0$ ou $\left.\operatorname{det}\left(\mathrm{d} g_{p}\right)<0\right)$, onde $A_{\mathrm{n}}^{*}$ e $A_{\mathrm{n}}$ sâo as aplicaçôes lineares auto-adjuntas associadas, respectivamente, às segundas formas fundamentais $I I_{\mathbf{n}}^{*}$. $\epsilon I I_{\mathrm{n}}$.

Corolário 1.1.1 Nas hipóteses e com as notaçôes da Proposiçâo 1.1.1 temos que

$$
\lambda_{i}^{*}=\frac{1}{\lambda_{i}} \quad \text { ou } \quad-\frac{1}{\lambda_{i}}, \quad i=1,2,
$$


onde $\lambda_{i}^{*} \in \lambda_{i}(i=1.2)$ sào. respectivamente, as curvaturas principais de $M^{*} \epsilon M \epsilon$ onde + ou - depende $d e \operatorname{det}\left(\mathrm{d} g_{p}\right)>0$ ou $\operatorname{det}\left(\mathrm{d} g_{p}\right)<0$.

Definição 1.1.1 A superfície $M^{*}$ da Proposiçào 1.1 .1 é denominada imagem esférica da superficie $M$.

Antes de prosseguirmos, esclarecemos que no restante desta seção o super-escrito * indicará elementos da geometria de $M^{*}$.

Um fato já conhecido, que decorre do Corolário 1.1.1 é que a classe das superfícies mínimas é invariante pela aplicaçào de Gauss em $S^{3}$. Mais precisamente, nas condiçòes da Proposição 1.1.1, temos o seguinte corolário.

Corolário 1.1.2 Se $M$ é uma superfície mínima em $S^{3}$ assim o é sua imagem esférica $M^{*}$.

Demonstração: $H^{*}=\frac{1}{2}\left(\lambda_{1}^{*}+\lambda_{2}^{*}\right)=-\frac{1}{2}\left(\frac{1}{\lambda_{1}}+\frac{1}{\lambda_{2}}\right)=-\frac{H}{K_{\epsilon}}$ de modo que, se $H=0$ entào $H^{*}=0$.

Este resultado nos leva naturalmente à seguinte questào:

Além das superfícies mínimas, existe outra classe de superfícies de Weingarten especiais em $S^{3}$ que seja invariante pela aplicação de Gauss?

Esta pergunta precisa de uma melhor reformulação. 
Seja $M$ uma superfície fechada em $S^{3}$, satisfazendo o seguinte: $H=f\left(H^{2}-K_{\epsilon}\right)$. $H^{2}-K_{\epsilon}>0$ e $K_{\epsilon}(p) \neq 0$ para todo $p$ em $M$. Podemos reformular a questào acima do seguinte modo:

Para que tipos de funçòes contínuas $f:[0,+\infty[\rightarrow \mathbb{R}$, reais analíticas em $] 0,+\infty[$. com $f(0)=0$. tem-se que a superficie $M$ c sua imagem esférica $M^{*}$ satisfazem. respectivamente, $H=f\left(H^{2}-K_{e}\right) \in H^{*}=f\left(H^{* 2}-K_{\epsilon}^{*}\right)$ ?

Antes de respondê-la façamos uma observação.

Observação 1.1.1 Nas condiçòes acima, temos sempre $K_{\epsilon}>0$ ou sempre $K_{\epsilon}<0$ sobre $M$. Se $K_{\epsilon}>0$, entào a curvatura de Gauss (intrínseca) de $M, K_{\text {int }}^{\prime}$, é positiva pois $K_{\text {int }}=1+K_{\epsilon}$. Portanto, pelo Teorema de Gauss-Bonnet, $M$ é homeomorfa à esfera. Nestas condições H. Hopf mostrou, em [Ho], que $M$ é totalmente umbílica, e portanto $M$ é uma esfera totalmente geodésica em $S^{3}$. Assim,devemos ter $K_{\epsilon}<0$ sobre $M$.

No que segue caracterizamos unicamente as classes das superfícies de Weingarten invariantes pela aplicação de Gauss no sentido da última questão apresentada. Para isto necessitamos do seguinte lema.

Lema 1.1.1 Seja $f:[0,+\infty[\rightarrow \mathbb{R}$ uma função contínua em sєu domínio, real analítica em $] 0,+\infty\left[, \operatorname{com} f(0)=0, f^{2}(x)<x, x \in\right] 0,+\infty[$. Entâo. $f$ é solução da equação funcional

$$
\left.f\left(\frac{x}{\left(f^{2}(x)-x\right)^{2}}\right)=-\frac{f(x)}{f^{2}(x)-x}, \quad x \in\right] 0 .+\infty[,
$$

se, e somente se, f é dada por

$$
f^{2}(x)=x-\sqrt{\frac{x}{\imath(x)}} .
$$


onde l : ]0. $+\infty[\rightarrow] 0 .+\infty[$. é uma funçào real analítica com as seguintes propriedades:

$$
\begin{aligned}
& \text { (i) } \left.\quad(x) \geq \frac{1}{x}, \quad x \in\right] 0,+\infty[. \\
& \text { (ii) } 101=i d_{0 .+x \mid}
\end{aligned}
$$

$\epsilon$, além disso, $f(\iota(x))$ e $f(x)$ têm mesmo sinal para todo $x \mathrm{em}] 0,+\infty[$.

Demonstração: Suponhamos que $f$ seja uma funçào definida por (1.2) e satisfazendo as condiçòes (i) e (ii). Temos então, por um lado, que

$$
\begin{aligned}
f^{2}\left(\frac{x}{\left(f^{2}(x)-x\right)^{2}}\right) & =f^{2}\left(\frac{x}{\frac{x}{\iota(x)}}\right) \\
& =f^{2}(\iota(x)) \\
& =\iota(x)-\sqrt{\frac{\iota(x)}{\iota(\iota(x))}} \\
& =\iota(x)-\sqrt{\frac{\iota(x)}{x}}
\end{aligned}
$$

e, por outro, que

$$
\begin{aligned}
\frac{f^{2}(x)}{\left(f^{2}(x)-x\right)^{2}} & =\frac{f^{2}(x)}{\frac{x}{\iota(x)}} \\
& =\frac{x-\sqrt{\frac{x}{\iota(x)}}}{\frac{x}{\iota(x)}} \\
& =\iota(x)-\sqrt{\frac{\iota(x)}{x}}
\end{aligned}
$$

de onde vemos que

$$
\left.f^{2}\left(\frac{x}{\left(f^{2}(x)-x\right)^{2}}\right)=\frac{f^{2}(x)}{\left(f^{2}(x)-x\right)^{2}}, \quad x \in\right] 0,+\infty[.
$$

Desde que $f(\iota(x))$ e $f(x)$ têm mesmo sinal, para todo $x$ positivo, a igualdade dada em (1.1) se verifica. 
Reciprocamente, suponhamos que $f$ seja uma funçào nas condiçòes dạdas e que seja soluçào da equação 1.1: Definamos uma funçào $\iota:] 0 .+\infty[\rightarrow] 0 .+\infty[$ por

$$
\iota(x)=\frac{x}{\left(f^{2}(x)-x\right)^{2}}
$$

Vejamos que a funçào, satisfaz as condiçòes que desejamos. Desde que

$$
f^{2}(x)-x<0
$$

e portanto,

$$
f^{2}(x)-2 x<0
$$

temos que

$$
f^{4}(x)-2 x f^{2}(x) \leq 0
$$

Assim,

$$
\left(f^{2}(x)-x\right)^{2}-x^{2} \leq 0
$$

de modo que obtemos

$$
\iota(x)=\frac{x}{\left(f^{2}(x)-x\right)^{2}} \geq \frac{1}{x}
$$

e isto demonstra que a função $\iota$ satisfaz (i). Agora, utilizando a definiçào de $\iota$ e o fato de $f$ ser soluçào da equaçào 1.1, obtemos a seguinte seqüência de igualdades:

$$
\begin{aligned}
(\iota \circ \iota)(x)=\iota(\iota(x)) & =\frac{\iota(x)}{\left(f^{2}(\iota(x))-\iota(x)\right)^{2}} \\
& =\frac{\frac{x}{\left(f^{2}(x)-x\right)^{2}}}{\left(\frac{f^{2}(x)}{\left(f^{2}(x)-x\right)^{2}}-\frac{x}{\left(f^{2}(x)-x\right)^{2}}\right)^{2}} \\
& =\frac{\frac{x}{\left(f^{2}(x)-x\right)^{2}}}{\left(\frac{f^{2}(x)-x}{\left(f^{2}(x)-x\right)^{2}}\right)^{2}} \\
& =\frac{\frac{x}{\left(f^{2}(x)-x\right)^{2}}}{\frac{1}{\left(f^{2}(x)-x\right)^{2}}} \\
& =x .
\end{aligned}
$$


Tendo definido 1 satisfazendo (i) e (ii), obtemos a expressào para $f$ dada na equaçào 1.2. De fato.

$$
\iota(x)=\frac{x}{\left(f^{2}(x)-x\right)^{2}}
$$

nos dá que

$$
\left(f^{2}(x)-x\right)^{2}=\frac{x}{l(x)}
$$

e, desde que $f^{2}(x)<x$, obtemos

$$
x-f^{2}(x)=\sqrt{\frac{x}{\iota(x)}} .
$$

Assim, acabamos de demonstrar o lema.

Observação 1.1.2 (a) O Lema 1.1.1 coloca o conjunto das soluçòes da equaçào 1.1 em correspondência bijetiva com o conjunto das involuções $\iota:] 0,+\infty[\rightarrow] 0,+\infty[$ tais que $\iota(x) \geq \frac{1}{x}$ para todo $\left.x \in\right] 0,+\infty[$. Observemos ainda que tais involuçòes satisfazem as seguintes propriedades:

(i) $\lim _{x \rightarrow 0^{+}} \iota(x)=+\infty$,

(ii) $\iota$ é monótona estritamente decrescente.

De fato, a propriedade dada em (i) decorre imediatamente da condição $\iota(x) \geq \frac{1}{x}$. Por sua vez, a propriedade (ii) decorre de $\ell$ ser uma involuçào e do item (i). Verificamos isto facilmente: derivando $(\iota \circ \iota)(x)=x$ obtemos que $\iota^{\prime}(x)$ nunca se anula e o item (i) nos faz concluir que $\iota^{\prime}(x)<0$ sempre.

(b)É importante lembrarmos que as hipérboles $\iota(x)=\frac{k}{x}, \quad k \geq 1$, sào exemplos naturais e simples de tais involuçòes. 
A figura que segue ilustra uma dessas hipérboles.

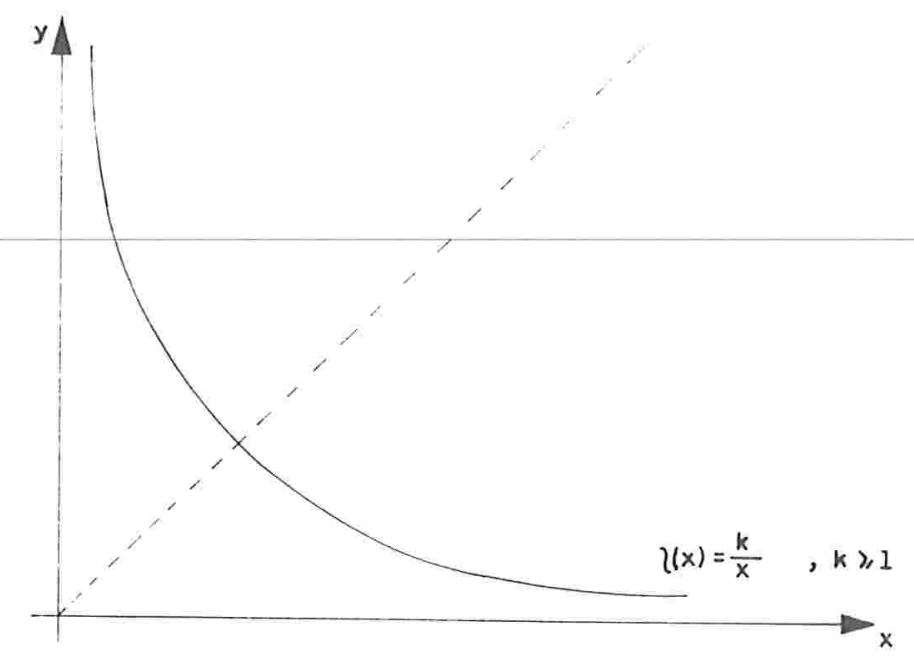

Figura 1

Proposição 1.1.2 Seja M uma superfície de Weingarten especial, fechada, orientável, conexa em $S^{3}$ e com imagem esférica $M^{*}$. M e $M^{*}$ pertencem a uma mesma classe de superfícies de Weingarten especiais se a funçâo $f$ que determina tal classe for uma das do tipo (1.2) do Lema 1.1.1.

Demonstração: Desde que $K_{e}(p)=\operatorname{det}\left(\mathrm{d} g_{p}\right)<0$ ( 1.1 .1$)$, pelo Corolário 1.1.1 temos que $\lambda_{i}^{*}=-\frac{1}{\lambda_{1}}, i=1,2$. Utilizando que

$$
H^{*}=-\frac{1}{2}\left(\frac{1}{\lambda_{1}}+\frac{1}{\lambda_{2}}\right)=-\frac{H}{K_{\epsilon}}
$$

e que

$$
H^{* 2}-K_{\epsilon}^{* *}=\frac{\left(\lambda_{1}-\lambda_{2}\right)^{2}}{4\left(\lambda_{1} \lambda_{2}\right)^{2}}=\frac{H^{2}-K_{\epsilon}}{K_{\epsilon}^{2}},
$$


obtemos que

$$
H^{*}=f\left(H^{* 2}-K_{\epsilon}^{*}\right)
$$

equivale a

$$
-\frac{H}{K_{\epsilon}}=f\left(\frac{H^{2}-K_{\epsilon}}{K_{\epsilon}^{2}}\right)
$$

Desde que $H=f\left(H^{2}-K_{\epsilon}\right)$, obtemos da última igualdade que

$$
f\left(\frac{H^{2}-K_{\epsilon}}{K_{\epsilon}^{2}}\right)=-\frac{f\left(H^{2}-K_{\epsilon}\right)}{K_{\epsilon}} .
$$

A equaçào funcional 1.1 do Lema 1.1 .1 é entào obtida fazendo $x=H^{2}-K_{\epsilon}$ e usando que $K_{\epsilon}=f^{2}\left(H^{2}-K_{\epsilon}\right)-\left(H^{2}-K_{\epsilon}\right)$. O resultado agora segue imediat amente do Lema 1.1.1.

Observação 1.1.3 (a) As classes das superfícies especiais obtidas na Proposiçào 1.1.2 incluem a das superfícies mínimas $\left(f \equiv 0\right.$ equivale à classe das $f$ obtidas $\operatorname{com} \iota(x)=\frac{1}{x}$ ). (b) Dentre as classes de superfícies obtidas destacamos aquelas definidas por $f(x)=$ $\pm c \sqrt{x}, \operatorname{com} c$ constante, $0<c<1$. Para cada $c$, uma tal classe é obtida tomando a involuçào $\iota(x)=\frac{k}{x}$, onde $k=\frac{1}{\left(1-c^{2}\right)^{2}}$ e podem ser caracterizadas como aquelas em que as superfícies $M$ e $M^{*}$ satisfazem a uma equação do tipo

$$
m \lambda_{1}+n \lambda_{2}=0, \quad m+n=2,
$$

também satisfeita pelas superfícies mínimas $(m=n=1)$. A saber, $M$ e $M^{*}$ satisfazem (1.5) $\operatorname{com} m=1-c, n=1+c$, no caso em que fé positiva, e. $m=1+c, n=1-c$, no caso em que f é negativa. Além disso, cada tal $f$ satisfaz a condiçào de elipticidade (veja [SaET1]):

$$
\left.4 x\left(f^{\prime}(x)\right)^{2}<1, \quad \text { para todo } x^{\prime} \text { em }\right] 0,+\infty[
$$


(c) Cada uma das classes de superfícies especiais obtidas na Proposiçào 1.1.2 incluem um único toro flat. Vejamos isto. Fixemos incialmente uma daquelas classes da Proposiçào 1.1.2. isto é, uma daquelas $f$ definidas por (1.2). Olhando para a definiçào de $f$, podemos escrever

$$
K_{\epsilon}=-\sqrt{\frac{x}{1(x)}}
$$

Como $K_{\epsilon}=\lambda_{1} \lambda_{2} \equiv-1$ ao longo de cada toro flat temos que $-1=-\sqrt{\frac{x}{\ell(x)}}$ de modo que $\iota(x)=x$.

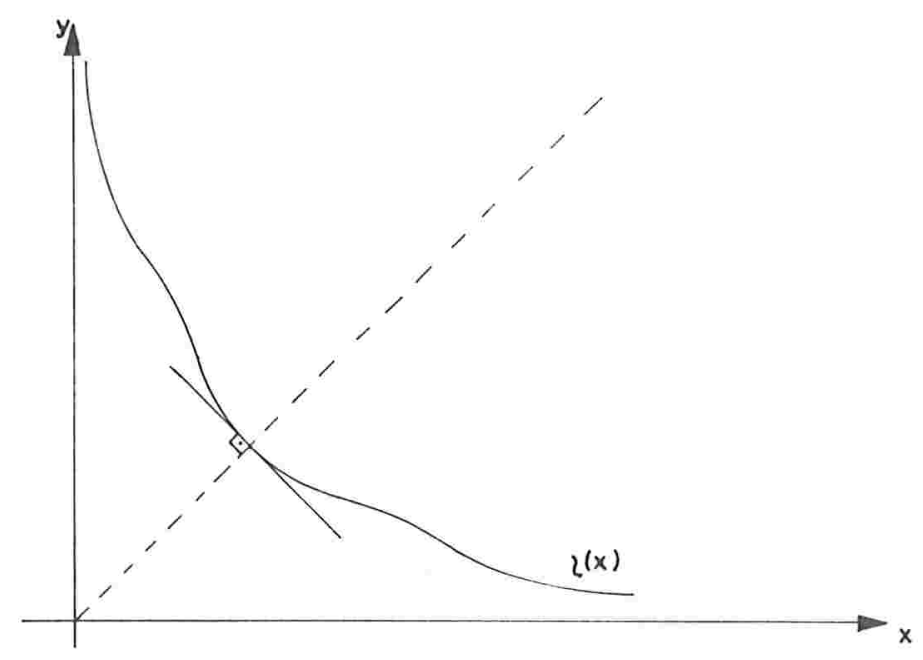

Figura 2

Assim, o único toro flat em cada tal classe corresponde ao único ponto $x \in] 0,+\infty[$ tal que $\iota(x)=x$ (lembremos que essa involução em $] 0,+\infty[$ intercepta a bissetriz do primeiro quadrante uma única vez, como ilustra a Figura 2).

Uma outra maneira de vermos isto é observando que os gráficos das funçòes $f$ e $g$, onde $f$ dá a fixada classe e $g$ é a funçào que dá a classe das superfícies especiais de Weingarten uma única vez. 
A Figura 3, abaixo, ilustra esta situaçào no caso em que $f(x)=c \sqrt{x}$ para alguns $0<c<1$.

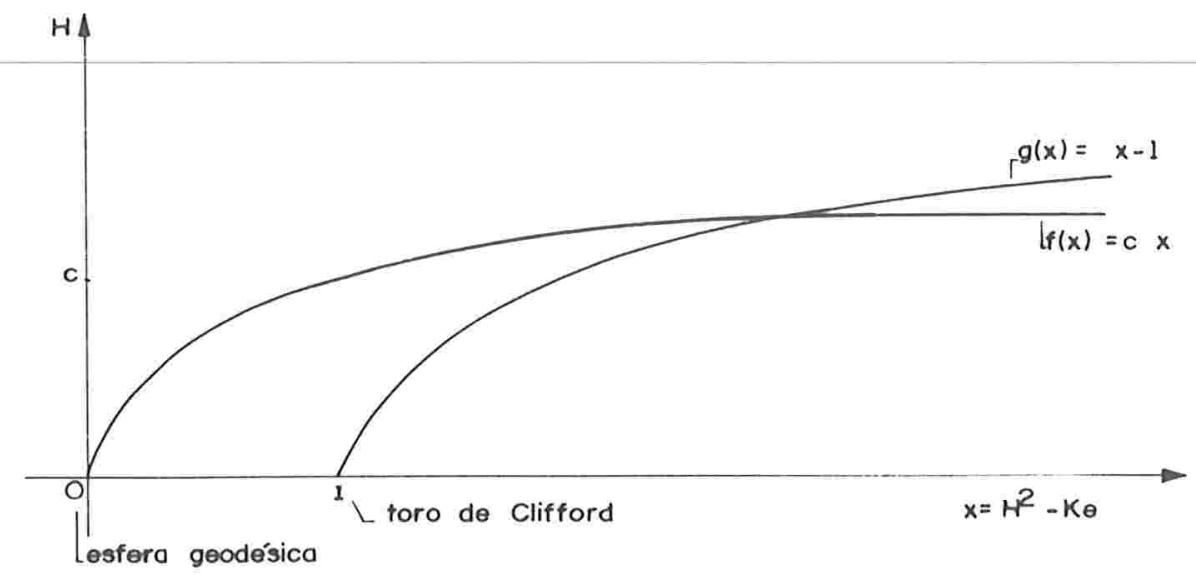

\section{Figura 3}

Apenas para enriquecermos um pouco mais esta discussào. observemos que no caso $f \equiv 0$ (superfícies mínimas) o toro flat que está na classe é o toro de Clifford. Façamos uma análise geométrica sobre esta observação, para o caso das classes das $f(x)=c \sqrt{x}$, $0<c<1$ (o caso $-1<c<0$ pode ser tratado analogamente). Estamos supondo que $\lambda_{1}>\lambda_{2}, \lambda_{1}>0$ sobre $M$. Desta forma, a equação 1.5 se reescreve como

$$
\epsilon_{c} \lambda_{1}+\left(2-\epsilon_{c}\right) \lambda_{2}=0
$$

onde $\epsilon_{c}>0$ é tal que $0<c=f(1)=1-\epsilon_{c}$. Então, $\lambda_{2}=-\frac{\epsilon_{c}}{2-\epsilon_{c}} \lambda_{1}$ e para cada $\epsilon_{c}$ temos que $\lambda_{1} \lambda_{2}=-1$ equivale à soluçào única de (1.6): $\lambda_{1} \equiv \sqrt{\frac{2-\epsilon_{c}}{\epsilon_{c}}}$ e $\lambda_{2} \equiv-\sqrt{\frac{\epsilon_{c}}{2-\epsilon_{c}}}$. Isto confirma a existência de um único toro flat em cada uma destas classes. 


\subsection{Sobre a EDO que determina a curva geradora de uma superfície de Weingarten especial rota- cional em $S^{3}$}

O objetivo principal desta seçào é a apresentaçào da EDO reduzida que rege a curva geradora de uma superfície de Weingarten especial rotacional em $S^{3}$. Conceituamos uma superfície rotacional pela propriedade de invariância por uma açào de um certo subgrupo $G$ do grupo das isometrias de $S^{3}$. Nada mais natural do que começarmos apresentando uma descriçào da geometria orbital de uma tal Gi-açào.

Lembremos que estamos considerando $S^{3}$ como sendo a seguinte hipersuperfície de $\mathbb{R}^{4}$ :

$$
S^{3}=\left\{\left(x_{1}, x_{2}, x_{3}, x_{4}\right) \in \mathbb{R}^{4} ; \sum_{i} x_{i}^{2}=1\right\} .
$$

No que segue, denotamos por $S^{2}(c)$ a 2-esfera euclideana de curvatura Gaussiana $c$, ou seja, a 2-esfera em $\mathbb{R}^{3}$ de raio $1 / \sqrt{c}$, e por $S^{1}(r)$ o círculo em $\mathbb{R}^{2}$ de raio $r$. Escrevemos simplesmente $S^{1}=S^{1}(1)$ e $S^{2}=S^{2}(1)$.

Seja $G$ o subgrupo das transformações isométricas de $S^{3}$ que deixam fixada, ponto a ponto, a geodésica dada por $x_{1}^{2}+x_{2}^{2}=1$. Em relaçào à base canônica $\left\{\epsilon_{1}, \epsilon_{2}, \epsilon_{3}, \epsilon_{4}\right\}$ de $\mathbb{R}^{4}$, cada matriz de um elemento $\sigma \in G$ pode ser escrita como

$$
\sigma=\left(\begin{array}{cc}
I & 0 \\
0 & \tilde{\sigma}
\end{array}\right)
$$

onde $I$ é a $2 \times 2$-matriz identidade e $\tilde{\sigma}$ é uma $2 \times 2$-matriz no grupo ortogonal $O(2)$. Desta forma, podemos pensar na $G$-ação sobre $S^{3}$ como numa $O(2)$-açào sobre $S^{3}$ nas duas últimas coordenadas. As órbitas desta açào sào de dois tipos: pontos fixos (órbitas de pontos do grande círculo fixado) e órbitas homeomorfas a $S^{1}$. Cada uma das do segundo tipo pode ser escrita como segue. Se a órbita passa por $\left(x_{1}, x_{2}, x_{3}, x_{4}\right) \in S^{3}$ 
$\operatorname{com} x_{1}^{2}+x_{2}^{2}<1$. entào ela é a interseç̧ào de $S^{3}$ com o plano afim em $\mathbb{R}^{4}$ passando por $\left(x_{1}, x_{2}, 0.0\right)$ e paralelo a $\{(0.0)\} \times \mathbb{R}^{2}$. sendo. portanto. do tipo $\varsigma^{1}(r) \operatorname{com} r=$ $\sqrt{1-x_{1}^{2}-x_{2}^{2}}=\sqrt{x_{3}^{2}+x_{4}^{2}}$. Por outro lado. consideremos o hemisfério fechado totalmente geodésico: $S_{+}^{2}=\left\{\left(x_{1}, x_{2}, x_{3}, 0\right) \in S^{3}: x_{3} \geq 0\right\}$. Toda órbita desta $G$-açào intercepta $S_{+}^{2}$ e. reciprocamente, cada ponto de $S_{+}^{2}$ está numa única órbita. Assim, identificamos $S_{+}^{2}$ com o espaço de órbitas $S^{3} / G$, desta $G$-açào, e definimos a projeçào canônica $\pi: S^{3} \rightarrow S_{+}^{2}$ por

$$
\pi\left(x_{1}, x_{2}, x_{3}, x_{4}\right)=\left(x_{1}, x_{2}, \sqrt{x_{3}^{2}+x_{4}^{2}}, 0\right) .
$$

Uma parametrizaçào de $S_{+}^{2}$ é dada a seguir. Denotemos por $o=(0,0,1,0)$ o centro de $S_{+}^{2}$. Para cada $p \in S_{+}^{2}$, seja $\rho(p)=d(p, o)$, onde $d$ é a distância riemanniana de $S^{3}$ e, se $p$ nào é o centro o, seja $\theta(p)$ o ângulo entre o segmento geodésico $\overline{o p}$ em $S_{+}^{2}$ ligando $o$ a $p$ e uma pré-fixada geodésica $\eta$ em $S_{+}^{2}$ passando por $o$ (veja Figura 4). Assim, associamos a cada $p \in S_{+}^{2}$ as coordenadas $(\theta(p), z(p))$. onde $z(p)=\cos \rho(p)$. Desta forma, se $p=\left(x_{1}, x_{2}, x_{3}, 0\right) \in S_{+}^{2}$, entào

$$
\begin{cases}x_{1}=\sqrt{1-z^{2}} \cos \theta & 0 \leq \theta<2 \pi \\ x_{2}=\sqrt{1-z^{2}} \operatorname{sen} \theta, & 0<z<1 . \\ x_{3}=z & \end{cases}
$$

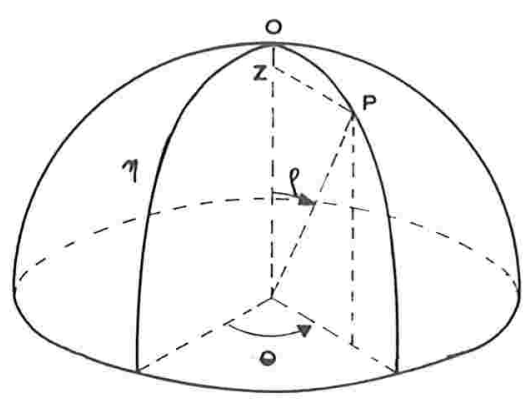

Figura 4 
Observação 1.2.1 A G-órbita passando por um ponto $p(z . \theta)$ dado por

$$
p(z . \theta)=\left(\sqrt{1-z^{2}} \cos \theta, \sqrt{1-z^{2}} \operatorname{sen} \theta . z, 0\right)
$$

pode ser escrita, por exemplo, por

$$
\left\{\left(\sqrt{1-z^{2}} \cos \theta \cdot \sqrt{1-z^{2}} \operatorname{sen} \theta, z \cos \varphi,-z \operatorname{sen} \varphi\right): \varphi \in[0,2 \pi]\right\} \cong S^{1}(z) .
$$

Desde que $S_{+}^{2}$ é totalmente geodésica e perpendicular a todas as $G$-órbitas, equipamos $S^{3} / G$ com a métrica da "distância orbital" ${ }^{1}$ como sendo a métrica da curvatura constante sobre $S_{+}^{2}$. Portanto, segundo o sistema de coordenadas acima definido, escrevemos

$$
\mathrm{d} s^{2}=\frac{\mathrm{d} z^{2}}{1-z^{2}}+\left(1-z^{2}\right) \mathrm{d} \theta^{2}
$$

A seguir, passamos ao estudo das superfícies $G$-invariantes em $S^{3}$.

Definição 1.2.1 Dizemos que uma superfície $M^{2} \hookrightarrow S^{3}$ é rotacional se $M^{2}$ é G-inva-

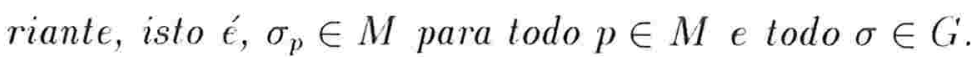

O estudo das superfícies rotacionais em $S^{3}$ pode ser reduzido ao estudo do comportamento de suas "curvas geradoras".

Definição 1.2.2 Seja $M$ uma superfície rotacional $\mathrm{em} S^{3}$, isto é, uma superfície $G$-invariante. A curva $\gamma=M / G \subset S_{+}^{2}$ é denominada curva geradora de $M$.

Antes de prosseguirmos, observemos que a pré-imagem, $\pi^{-1}(\gamma)$, em $S^{3}$, de uma curva $\gamma$ é topologicamente um toro $S^{1} \times S^{1}$ imerso se $\gamma$ é uma curva fechada com auto-interseç̧ôes. Se $\gamma$ é fechada e simples, então $\pi^{-1}(\gamma)$ é topologicamente um mergulho do toro $S^{1} \times S^{1}$.

\footnotetext{
${ }^{1} \mathrm{Um}$ tratamento geral sobre a estrutura métrica de um espaço de órbitas se encontra em [BdCH] e $[\mathrm{HL}]$.
} 
e no caso em que $\gamma$ é uma curva simples com pontos inicial e final no bordo de $S_{+}^{2}$. entào $\pi^{-1}(\jmath)$ é topologicamente um mergulho de $S^{2}$ (Figura 5).

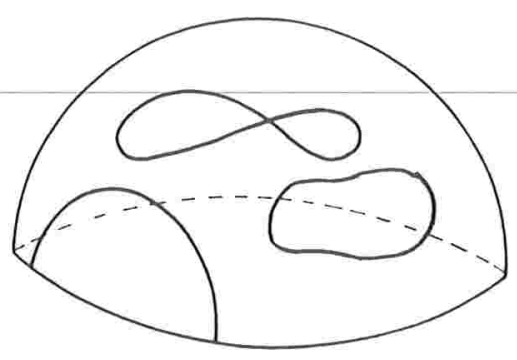

Figura 5

Seja $\gamma(s)=(\theta(s), z(s))$ uma curva parametrizada pelo comprimento de arco, ou seja, $\frac{\mathrm{d} \gamma}{\mathrm{d} s}=(\dot{\theta}, \dot{z})$ é tal que

$$
\frac{\dot{\sim}^{2}}{1-z^{2}}+\dot{\theta}^{2}\left(1-z^{2}\right)=1
$$

Sejam $\mathbf{e}_{\theta}=\frac{1}{\sqrt{1-z^{2}}} \frac{\partial}{\partial \theta}, \quad \mathbf{e}_{z}=-\sqrt{1-z^{2}} \frac{\partial}{\partial z}$, e seja a oângulo entre $\frac{\mathrm{d} \gamma}{\mathrm{d} s} \mathrm{e} \mathbf{e}_{z}$, como ilustra a Figura 6 abaixo. Então

$$
\begin{aligned}
\cos \alpha & =\frac{\dot{z}}{\sqrt{1-z^{2}}}, \\
\operatorname{sen} \alpha & =\dot{\theta} \sqrt{1-z^{2}} \mathrm{e} \\
\frac{\mathrm{d} \alpha}{\mathrm{d} s} & =\frac{\ddot{z}}{\theta\left(1-z^{2}\right)}+\frac{z \dot{z}^{2}}{\dot{\theta}\left(1-z^{2}\right)^{2}} .
\end{aligned}
$$

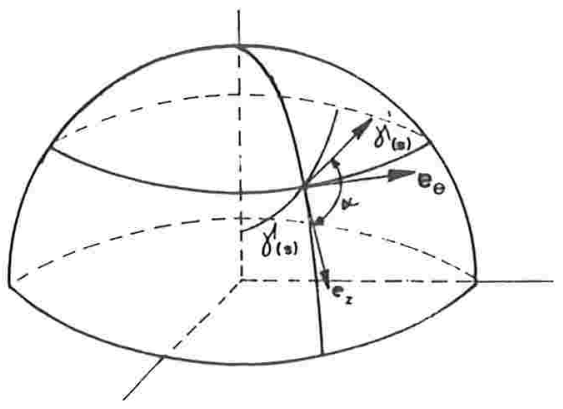

Figura 6 
Ainda. seja $\mathrm{n}=\cos \alpha \mathrm{e}_{\theta}-\operatorname{sen} \alpha \mathrm{e}_{z}$. isto é.

$$
\mathbf{n}=-\frac{\dot{z}}{1-z^{2}} \frac{\partial}{\partial \theta}+\dot{\theta}\left(1-z^{2}\right) \frac{\partial}{\partial z}
$$

O vetor n é o vetor unitário normal a $\hat{\imath}$ em $\gamma(s)$.

A fórmula de Liouville para curvatura geodésica, $k_{g}(s)$, de $\gamma(s)$, é a seguinte:

$$
k_{g}=\frac{\mathrm{d} \alpha}{\mathrm{d} s}+k_{g_{1}} \cos \alpha+k_{g_{2}} \operatorname{sen} \alpha
$$

onde $k_{g_{1}}$ e $k_{g_{2}}$ sào, respectivamente, as curvaturas geodésicas do meridiano $\theta=$ constante e do paralelo $z=$ constante. Assim, como $k_{g_{1}} \equiv 0$ e $k_{g_{2}}=\frac{z}{\sqrt{1-z^{2}}}$, obtemos

$$
k_{g}(s)=\frac{\mathrm{d} \alpha}{\mathrm{d} s}+\frac{z}{\sqrt{1-z^{2}}} \operatorname{sen} \alpha,
$$

ou seja,

$$
k_{g}=\frac{\ddot{z}\left(1-z^{2}\right)+z \dot{z}^{2}}{\dot{\theta}\left(1-z^{2}\right)^{2}}+z \dot{\theta}
$$

Agora, se $M$ é uma superfície rotacional de $S^{3}$ gerada por $\gamma(s)=(\theta(s), z(s))$, nào é difícil vermos que a aplicação linear auto-adjunta $A_{\mathrm{n}}$ associada à segunda forma fundamental, $I I_{\mathbf{n}}$, de $M$ em $\gamma(s)$, tem somente dois autovalores distintos correspondentes a dois subespaços invariantes irredutíveis do espaço tangente $T_{\gamma(s)} M$. Um dos subespaços é tangente à curva $\gamma$ em $\gamma(s)$ e o outro é tangente à órbita $G(\gamma(s)) \cong S^{1}$. Portanto, uma das curvaturas principais de $M$ em $\gamma(s)$, digamos $\lambda_{1}$, é a curvatura geodésica $k g$ dada em (1.8). Para obtermos o valor da outra curvatura principal, $\lambda_{2}$, de $M$ em $\gamma(s)$, inicialmente consideremos o volume funcional, $v(s)$, da $G$-órbita no ponto $\gamma(s)$. Este é dado por $v(s)=2 \pi z(s)$, de modo que

$$
\begin{aligned}
\frac{\partial}{\partial \mathrm{n}}(\ln v) & =\langle\operatorname{grad}(\ln z) \cdot \mathrm{n}\rangle \\
& =\left\langle 0 \mathrm{e}_{\theta}-\frac{\sqrt{1-z^{2}}}{z} \mathrm{e}_{z}, \cos \alpha \mathrm{e}_{\theta}-\operatorname{sen} \alpha \mathbf{e}_{z}\right\rangle \\
& =\frac{\sqrt{1-z^{2}}}{z} \operatorname{sen} \alpha .
\end{aligned}
$$


Além disso. utilizemos a seguinte fórmula para a curvatura média, $H=\frac{1}{2}\left(\lambda_{1}+\lambda_{2}\right)$. de.$M$ em $\gamma(s)$ (veja $[\mathrm{BdCH}]$ )

$$
H=\frac{1}{2}\left(k g-\frac{\partial}{\partial \mathrm{n}} \ln v\right)
$$

onde $v$ é o volume funcional da $G$-órbita no ponto $\gamma(s)$.

Estabelecemos tais consideraçòes na seguinte

Proposição 1.2.1 As curvaturas principais de uma superfície rotacional $M \hookrightarrow S^{3}$, tendo $\gamma(s)=(\theta(s), z(s))$ como curva geradora, sào dadas por

$$
\left\{\begin{array}{l}
\lambda_{1}=k g=\frac{\mathrm{d} a}{\mathrm{~d} s}+\frac{z}{\sqrt{1-z^{2}}} \operatorname{sen} \alpha, \\
\lambda_{2}=-\frac{\sqrt{1-z^{2}}}{z} \operatorname{sen} \alpha,
\end{array}\right.
$$

onde a é o ângulo cntre $\frac{\mathrm{d} \gamma}{\mathrm{d} s} \in \mathrm{e}_{\mathrm{Z}}$.

Podemos reescrever (1.10) por:

$$
\left\{\begin{array}{l}
\lambda_{1}=k g=\frac{\ddot{z}\left(1-z^{2}\right)+z \dot{z}^{2}}{\dot{\theta}\left(1-z^{2}\right)^{2}}+z \dot{\theta}, \\
\lambda_{2}=-\frac{\left(1-z^{2}\right) \dot{\theta}}{z} .
\end{array}\right.
$$

Finalmente, podemos apresentar a equaçào diferencial ordinária de segunda ordem. que só depende da coordenada z, e que é satisfeita pela curva geradora de uma superfície de Weingarten especial rotacional em $S^{3}$.

Proposição 1.2.2 Seja M uma superfície especial de Weingarten rotacional da classe de uma funçào contínua $f:[0,+\infty[\rightarrow \mathbb{R}, \operatorname{com} f(0)=0$ e real analítica $\mathrm{\epsilon m}] 0,+\propto[$. Então 
a curva $\gamma=M / G \mathrm{em} S_{+}^{2}$, parametrizada pelo comprimento de arco. satisfaz a seguinte equaçâo diferencial ordinária

$$
\tilde{z} \ddot{z}+z^{2}-\left(1-z^{2}-\dot{z}^{2}\right)= \pm 2 z \sqrt{1-z^{2}-\dot{z}^{2}} f\left(\frac{\left(z \ddot{z}+z^{2}+\left(1-z^{2}-\dot{z}^{2}\right)\right)^{2}}{4 z^{2}\left(1-z^{2}-\dot{z}^{2}\right)}\right) .
$$

onde + ou - depende, respectivamente, de $\dot{\theta}>0$ ou $\dot{\theta}<0, f \dot{\theta}= \pm \frac{\sqrt{1-z^{2}-\dot{z}^{2}}}{1-z^{2}}$. $0<z<1$.

Demonstração: Sendo $M$ uma superfície de Weingarten especial, então $M$ satisfaz a equaçào

$$
H=f\left(H^{2}-K_{\epsilon}\right)
$$

onde $H$ e $K_{e}$ sào as funçòes simétricas das curvaturas principais, $\lambda_{1}, \lambda_{2}$, dadas por

$$
H=\frac{1}{2}\left(\lambda_{1}+\lambda_{2}\right) \quad \text { e } \quad K_{\epsilon}=\lambda_{1} \lambda_{2}
$$

Portanto, podemos reescrever (1.13), em termos de $\lambda_{1}$ e $\lambda_{2}$, por

$$
\frac{1}{2}\left(\lambda_{1}+\lambda_{2}\right)=f\left(\frac{\left(\lambda_{1}-\lambda_{2}\right)^{2}}{4}\right) \text {. }
$$

Substituindo, diretamente na última equaçào as expressòes de $\lambda_{1}$ e $\lambda_{2}$ dadas em (1.11) e utilizando a expressào de $\dot{\theta}$ dada por $\dot{\theta}= \pm \frac{\sqrt{1-z^{2}-\dot{z}^{2}}}{1-z^{2}}$, obtida da condição de $\gamma(s)$ ser parametrizada pelo comprimento de arco, obtemos a equaçào diferencial ( 1.12 ). 


\title{
Superfícies especiais de tipo mínimo
}

\author{
$\operatorname{em} S^{3}$
}

Nosso objetivo principal neste capítulo é conceituar "classes de superfícies de tipo mínimo em $S^{3}$ " e mostrar que existem exemplos, imersos em $S^{3}$, de superfícies "tóricas" homeomorfas a $S^{1} \times S^{1}$.

\subsection{Conceituação}

Na Seção 1.1 do Capítulo 1 introduzimos as classes de superfícies de Weingarten especiais que são invariantes pela aplicaçào de Gauss em $S^{3}$. Tais classes invariantes são, a saber, aquelas dadas por funçôes $f:\left[0,+\infty\left[\rightarrow \mathbb{R}\right.\right.$ satisfazendo $f^{2}(x)=x-\sqrt{\frac{x}{\iota(x)}}$, onde as funçôes $\iota:] 0,+\infty[\rightarrow] 0,+\infty\left[\right.$ são involuções tais que $\left.\iota(x) \geq \frac{1}{x}, x \in\right] 0,+\infty[$. Chamamos atenção novamente para o fato de que se $\iota(x)=\frac{1}{x}$ para todo $x$ em $] 0,+\infty[$, entào $f \equiv 0$, ou seja, a classe das superfícies mínimas é uma que satisfaz tal invariância.

A seguir, enumeramos mais alguns fatos que mostram certas analogias entre cada uma dessas classes de superfícies especiais obtidas e a classe das superfícies mínimas. 
1. $f(0)=0$. ou seja. as 2-esferas totalmente geodésicas sào superfícies especiais em cada uma dessas tais classes.

2. Sobre cada superfície $M$, numa dessas classes, a curvatura de Gauss-Kronecker. $K_{t}$, é negativa. De fato:

$$
\begin{aligned}
K_{\epsilon} & =H^{2}-\left(H^{2}-K_{\epsilon}\right) \\
& =\left(f\left(H^{2}-K_{\epsilon}\right)\right)^{2}-\left(H^{2}-K_{\epsilon}\right) \\
& =H^{2}-K_{\epsilon}-\frac{\sqrt{H^{2}-K_{\epsilon}}}{\iota\left(H^{2}-K_{\epsilon}\right)}-\left(H^{2}-K_{\epsilon}\right) \\
& =-\sqrt{\frac{H^{2}-K_{\epsilon}}{\iota\left(H^{2}-K_{\epsilon}\right)}}<0 .
\end{aligned}
$$

3. Em cada uma dessas classes existe um único toro flat mergulhado em $S^{3}$.

Levando em conta os fatos acima relacionados, damos a seguinte definição.

Definição 2.1.1 Denominamos as classes de superfícies especiais em $S^{3}$ dadas pelas funções $f$ obtidas no Lema 1.1.1 do Capítulo 1, de classes de superfícies especiais de tipo mínimo em $S^{3}$.

O objetivo principal deste capítulo é garantir a existência de superfícies compactas, homeomorfas a $S^{1} \times S^{1}$, em cada uma das classes das superfícies de tipo mínimo. Podemos formalizar isto no seguinte resultado, o qual já foi enunciado na introdução deste trabalho.

Teorema A Em cada classe de superfícies especiais de tipo mínimo, existe uma quantidade infinita de superfícies compactas de gênero um, imersas em $S^{3}$. 


\subsection{Demonstração do Teorema A}

A procura de exemplos mergulhados de superfícies compactas de gênero um em $S^{3}$ nos leva. naturalmente, ao estudo das superfícies rotacionais de tipo mínimo em $S^{3}$. A existência, ou nào. de outros exemplos mergulhados de superfícies compactas homeomorfas ao toro $S^{1} \times S^{1}$, além do único toro flat na classe (veja $(1.1 .3(\mathrm{c}))$ ), é uma questào ainda sem resposta.

Superfícies de Weingarten especiais rotacionais, em $S^{3}$, foram introduzidas de maneira geral na Seçào 1.2 do Capítulo 1. Obtivemos, lá, a equaçào diferencial satisfeita por uma curva geradora de uma tal superfície, segundo a parametrizaçào do espaço de órbitas, $S_{+}^{2}$, dada por

$$
\begin{cases}x_{1}=\sqrt{1-z^{2}} \cos \theta & 0 \leq \theta<2 \pi \\ x_{2}=\sqrt{1-z^{2}} \operatorname{sen} \theta, & 0<z<1 . \\ x_{3}=z & \end{cases}
$$

Ressaltamos que a equaçào diferencial (1.12) na Proposiçào 1.2.2, foi obtida segundo a parametrizaçào acima, a partir da equação

$$
\frac{\lambda_{1}+\lambda_{2}}{2}=f\left(\frac{\left(\lambda_{1}-\lambda_{2}\right)^{2}}{4}\right)
$$

onde, $\lambda_{1}$ e $\lambda_{2}$ sào as curvaturas principais da superfície $M$ que satisfaz a mesma e tal que $f:[0 .+\infty[\rightarrow \mathbb{R}$ é uma função contínua, com $f(0)=0$ e real analítica em $] 0,+\infty[$. Lembramos que no caso particular das superfícies de tipo mínimo, cada $f$ satisfaz $f^{2}(x)=$ $x-\sqrt{\frac{x}{\iota(x)}}$, sendo que a involução ı é tal que $\iota(x) \geq \frac{1}{x}$ e $\operatorname{com} f(\iota(x)) f(x) \geq 0$.

Fazendo $f$, na equação 2.1, como uma das dos tipos acima consideradas, obtemos após alguns cálculos diretos, a seguinte equação:

$$
,\left(\frac{\left(\lambda_{1}-\lambda_{2}\right)^{2}}{4}\right)=\frac{\left(\lambda_{1}-\lambda_{2}\right)^{2}}{4 \lambda_{1}{ }^{2} \lambda_{2}{ }^{2}} .
$$


Utilizando esta última equaçào do mesmo modo como fizemos na demonstraçào da Proposiçào 1.2.2. obtemos uma equaçào diferencial satisfeita pela curva geradora, $\gamma$. de uma superfície em questào.

Proposição 2.2.1 Seja M uma superfície especial rotacional. completa, de tipo mínimo em $S^{3}$, na classe de uma funçâo f como no Lema 1.1.1 do Capítulo 1. Entào a curva $\gamma=M / G$ em $S_{+}^{2}=S^{3} / G$, parametrizada pelo comprimento de arco, satisfaz a seguinte equaçào diferencial ordinária:

$$
,\left(\frac{\left(z \ddot{z}+z^{2}+\left(1-z^{2}-\dot{z}^{2}\right)\right)^{2}}{4 z^{2}\left(1-z^{2}-\dot{z}^{2}\right)}\right)=\frac{\left(z \ddot{z}+z^{2}+\left(1-z^{2}-\dot{z}^{2}\right)\right)^{2}}{4(\ddot{z}+z)\left(1-z^{2}-\dot{z}^{2}\right)} .
$$

Demonstração: Basta substituirmos as expressòes de $\lambda_{1}$ e $\lambda_{2}$ dadas na equação 1.11 do Capítulo 1 na equação 2.2 e utilizarmos a condição de $\gamma$ ser parametrizada pelo comprimento de arco.

Observação 2.2.1 (a) Outra maneira de obtermos a equação 2.3 é substituirmos a expressão que define a funçào $f$ na equação diferencial 1.12 do Capítulo 1 .

(b) As únicas soluções passando pelo centro $o=(0,0,1,0)$ de $S_{+}^{2}$ sào do tipo $\theta=$ constante, ou seja, são os semi-círculos totalmente geodésicos ortogonais ao bordo de $S_{+}^{2}$ (veja Figura 7b). As superfícies correspondentes a esses grandes círculos ortogonais ao bordo são as 2-esferas totalmente geodésicas de $S^{3}$. Elas são isoparamétricas com $\lambda_{1} \equiv \lambda_{2} \equiv 0$ e, portanto, satisfazem a equaçào 2.1: $f(0)=0$.

(c) De acordo com a Observaçào 1.1.3 do Capítulo 1, sabemos que existe uma única solução do tipo $z=$ constante, correspondente ao único toro flat na fixada classe (veja 
Figura $7 \mathrm{a}$ ). como já sabemos. o único toro flat corresponde ao único $\bar{x} \in] 0 .+\infty[$, tal que $\iota(\bar{x})=\bar{x}$. Assim, para simplificar notaçào, denotemos $\frac{1}{\bar{x}}$ por a . Assim obtemos um único $\tilde{z}_{a} .0<\tilde{z}_{a}<1$. tal que $\tilde{z}_{a}=\sqrt{\frac{1}{2}+\frac{1}{2} \sqrt{1-a}}$. Isto nào é difícil de ser visto. desde que se $z \equiv$ constante, entào $\dot{\sim} \equiv 0$ e $\ddot{z} \equiv 0$, de modo que, pela equaçào 2.3 . obtemos $a=4 z^{2}\left(1-z^{2}\right)$.

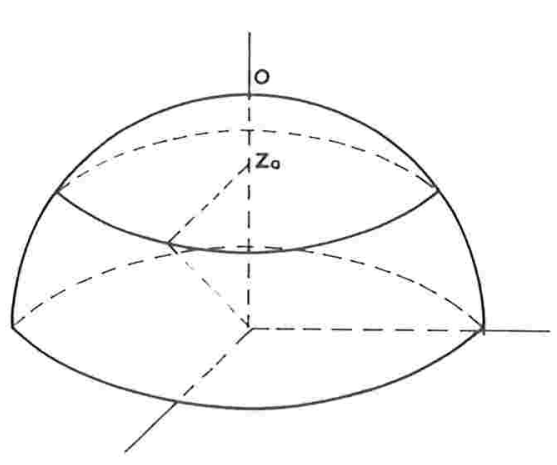

Figura 7a

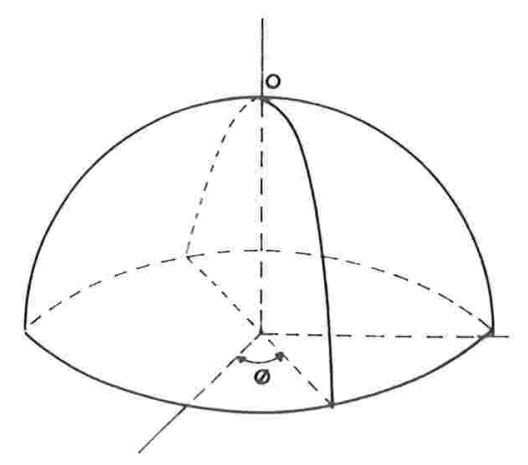

Figura $7 b$

No que segue, considere fixada uma classe de superfícies de tipo mínimo, ou seja, uma das $f$ do Lema 1.1 .1 está fixada.

Com o propósito de evitarmos cansativas repetições, referir-nos- emos a uma solução da equaçào diferencial em questào simplesmente por "curva soluçào".

Primeiramente, a equação 2.3 pode ser reescrita por

$$
G(x)=\iota(x)-\frac{x}{f^{2}(x)-x}=0
$$

onde $x(s, z, \dot{z}, \ddot{z})=\frac{\left(z \ddot{z}+z^{2}+\left(1-z^{2}-\dot{z}^{2}\right)\right)^{2}}{4 z^{2}\left(1-z^{2}-\dot{z}^{2}\right)}$. Assim a equação 2.3 se escreve implicitamente por

$$
F(s, z, \dot{z}, \ddot{z})=G(x(s, z, \dot{z}, \ddot{z}))=0
$$


Seja $R=\left\{(s, z . u, v) \in \mathbb{R}^{4}: \quad s \in \mathbb{R}, \quad 0<z<1 .|u| \leq \sqrt{1-z^{2}} . v \neq-z\right\} \quad \subset \mathbb{R}^{4}$. As funçòes $F, \frac{\partial F}{\partial s} \cdot \frac{\partial F}{\partial z} \cdot \frac{\partial F}{\partial u} \mathrm{e} \frac{\partial F}{\partial u}$ sào contínuas em $R$.

De fato, $\frac{\partial F}{\partial s}=0, \frac{\partial F}{\partial z}=G^{\prime}(x) \frac{\partial x}{\partial z} \cdot \frac{\partial F}{\partial u}=G^{\prime}(x) \frac{\partial x}{\partial u} \cdot \frac{\partial F}{\partial x}=G^{\prime}(x) \frac{\partial x}{\partial x}$.

onde

$$
\begin{gathered}
G^{\prime}(x)=\iota^{\prime}(x)\left(1+\frac{1}{2} \sqrt{\frac{x}{\left(x^{\prime}\right)}}\right)+\frac{1}{2} \sqrt{\frac{(x)}{x}} \\
\frac{\partial x}{\partial z}=\frac{\left(z u+1-u^{2}\right)\left(z^{3} v-1+2 z^{2}+u^{2}\left(1-2 z^{2}-u^{2}\right)\right)}{2 z^{3}\left(1-z^{2}-u^{2}\right)^{2}}, \\
\frac{\partial x}{\partial u}=\frac{-u\left(z v+1-u^{2}\right)\left(1-u^{2}-2 z^{2}-z v\right)}{2 z^{2}\left(1-z^{2}-u^{2}\right)^{2}}
\end{gathered}
$$

onde as funçòes acima estào todas calculadas numa vizinhança de um ponto $P_{0}=\left(s_{0}, z_{0}, u_{0}, v_{0}\right) \in R$. Além disso, $G^{\prime}(x) \neq 0$, pois caso contrário teríamos que

$$
\iota^{\prime}(x)=-\frac{1}{2} \sqrt{\frac{l(x)}{x}}\left(1+\frac{1}{2} \sqrt{\frac{x}{\iota(x)}}\right)^{-1}
$$

de onde obteríamos que

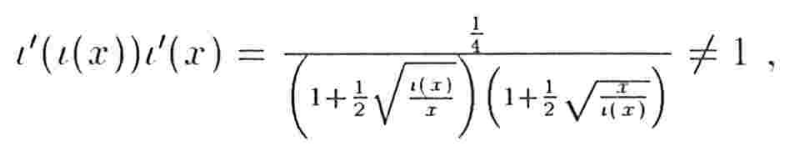

o que contradiria o fato de $\iota(\iota(x))=x$. Esta última condição mais a de que $\frac{\partial x}{\partial v} \neq 0$ (pois $x \neq 0$ ), nos dá que $\frac{\partial F}{\partial v} \neq 0$. Pela teoria das funções implícitas, isto é suficiente para garantirmos que, numa vizinhança próxima de $\left(s_{0}, z_{0}, \dot{z}_{0}, \ddot{z}_{0}\right) \in R$, onde $z_{0}=z\left(s_{0}\right)$, $\dot{z}_{0}=\dot{z}\left(s_{0}\right)$ e $\quad \ddot{z}_{0}=\ddot{z}\left(s_{0}\right)$, podemos explicitar localmente $\ddot{z}$ como uma funçào contínua de $s, z$ e $\dot{\sim}$.

Assim, pelo Teorema de Existência e Unicidade para equaçòes diferenciais ordinárias 
de segunda ordem. dado $P_{0}=\left(s_{0}, \tilde{z}_{0}, \dot{\sim}_{0}, \ddot{z}_{0}\right) \in R$. existe uma única soluçào local $\tilde{z}(s)$ da equaçào 2.3 satisfazendo. $\quad \tilde{z}\left(s_{0}\right)=z_{0} . \quad \dot{\tilde{z}}\left(s_{0}\right)=\dot{z}_{0}$. Seja $z(s)$ a continuaçào analítica de $\tilde{z}(s)$ e seja $\gamma(s)=(\theta(s), z(s)) . s \in \mathbb{R} \quad$ a curva soluçào, parametrizada pelo comprimento de arco, passando por $\left(\theta_{0}, \tilde{z}_{0}\right)$, com $\theta_{0}=\theta\left(s_{0}\right)$ e tal que $\dot{z}\left(s_{0}\right)=\dot{z}_{0}$.

Observemos que a curva soluçào nào pode permanecer constante em qualquer subintervalo de $\mathbb{R}$, pois se isto ocorresse teríamos $\dot{\theta} \equiv 0$ e $\dot{z} \equiv 0$, simultaneamente nesse subintervalo, o que seria uma contradição com o fato da curva ser parametrizada pelo comprimento de arco. Além disso, pelo Teorema de unicidade de soluçôes, nenhuma outra curva soluçào pode tangenciar qualquer soluçào do tipo $\theta \equiv$ constante. Devemos observar ainda que nenhuma curva soluçâo toca o bordo, exceto ortogonalmente, como o fazem todos os "grandes semi-círculos" ortogonais ao bordo. A técnica utilizada para demonstrar tais fatos é semelhante à utilizada por W. Y. Hsiang e B. L. Lawson em [HL] no estudo de hipersuperfícies fechadas mínimas de $S^{n}$. Técnica semelhante, porém em situaçòes diferentes das nossas, que podem servir como referência são encontradas no trabalho de B. Charlet, em [Ch].

Tendo em vista os fatos acima, se a curva solução, $\gamma=(\theta, z)$ não é um grande círculo, temos que $\dot{\theta}>0$ sempre, ou $\dot{\theta}<0$ sempre. Assim todas as curvas soluçòes podem ser expressas como $\gamma(\theta)=(\theta, z(\theta))$, onde $\theta$ percorre todos os números reais.

Para demonstrarmos o principal resultado deste capítulo (Teorema A) devemos ainda estabelecer mais alguns resultados.

Definição 2.2.1 Dizemos que $\theta_{0} \in \mathbb{R}$ é um ponto crítico de uma curva $\gamma(\theta)=(\theta, z(\theta))$ em $S_{+}^{2}$ se $\dot{z}(\theta)=0$. 
Lema 2.2.1 Seja $\gamma(\theta)=(\theta, z(\theta))$ uma curva soluçào de (2..3) e suponhamos que $\theta_{0}$ seja

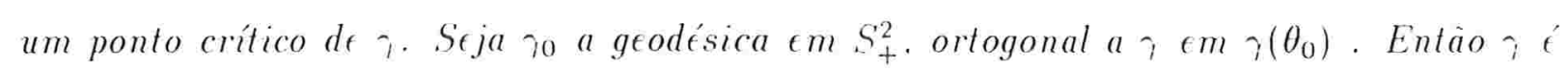
invariante pela reflexào $\mathrm{em}, S_{+}^{2}$ com relaçào a $\gamma_{0}$.

Demonstração: Sem perda de generalidade podemos supor $\theta_{0}=0$. Seja $\tau$ a reflexào em torno de $\gamma_{0}$. Sejam $\left.\tilde{\gamma}(\theta)\right)=\tau(\gamma(\theta))=(-\theta, z)$ e $\bar{\gamma}(\theta)=\gamma(-\theta)$. Obviamente. $\tilde{i}$ e $\bar{\gamma}$ sào curvas soluçòes e $\tilde{\gamma}(0)=\bar{\gamma}(0)$ e $\dot{\tilde{\gamma}}_{0}=\dot{\bar{\gamma}}_{0}$. Assim, pelo Teorema de unicidade para equaçòes diferenciais ordinárias de segunda ordem, temos que $\tilde{\gamma}=\bar{\gamma}$.

A Figura 8 ilustra a reflexào de uma curva $\gamma$ com relaçào a $\gamma_{0}$.

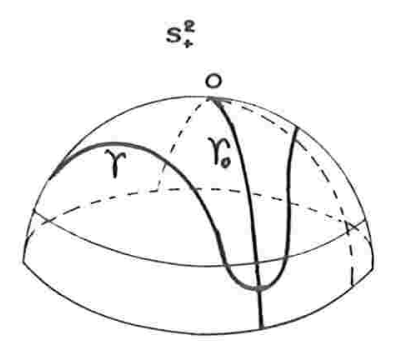

\section{Figura 8}

Definição 2.2.2 Dizemos que uma curva soluçâo $\gamma(\theta)=(\theta, z(\theta))$ é periódica se cxiste um número $p>0$, tal que $z(\theta+p)=z(\theta)$, para todo $\theta \mathrm{em} \mathbb{R}$.

Corolário 2.2.1 Uma curva soluçâo com pelo menos dois pontos críticos é periódica.

Demonstração: É uma consequëncia imediata do Lema 2.2.1 . 
Lema 2.2.2 Seja $\gamma(\theta)=(\theta . z(\theta))$ uma soluçào da equaçào 2.3 que intercepta o paralelo correspondente ao toro flat na classe. Então ’ é uma curéa periódica em $S_{+}^{2}$.

Demonstração: Vimos anteriormente que o único paralelo que é uma curva soluçào é dado por $z \equiv z_{a}=\sqrt{\frac{1}{2}+\frac{1}{2} \sqrt{1-a}}$,onde $a=\frac{1}{\bar{x}}$ e $\bar{x}$ é tal que $\iota(\bar{x})=\bar{x}$. Seja $\gamma(\theta)=(\theta, z(\theta))$ uma curva que intercepta $z \equiv \tilde{z}_{a}$. Se demonstrarmos que $\gamma$ atinge um máximo e um mínimo, podemos estabelecer a propriedade por reflexões em relaçào aos meridianos $\theta \equiv$ constante, conforme nos garante o Lema 2.2.1 (veja Figura 9).

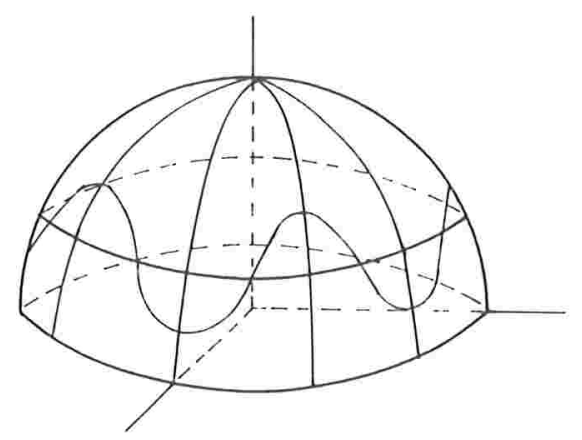

Figura 9

Seja $\theta_{0}$ tal que $z\left(\theta_{0}\right)=z a$. Se $z=z(\theta)$ nào possui máximo então, sem perda de generalidade, podemos supor que $z$ é monótona crescente a partir de $\theta_{0}$. Então, ou a curva $\gamma$ espirala em torno do paralelo $z \equiv z_{1}$, para algum $z_{1}$ constante, $z_{a}<z_{1}<1$ (veja Figura 10a), ou, $\gamma$ espirala em torno do "centro" o de $S_{+}^{2}$.

Se ocorresse o primeiro caso, por dependência contínua das soluçòes com relação às condições iniciais, o paralelo em questão seria uma curva solução, o que seria um contradiçào, pois teríamos um outro toro flat na classe! Se ocorresse o segundo caso e tomássemos uma curva soluçào $\bar{\gamma}$, muito próxima de algum meridiano que tangenciasse a curva ? num ponto muito próximo do "centro" o (veja Figura 10b), entào, por 
unicidade. $\bar{\imath}$ e $\hat{\imath}$ deveriam coincidir. Contradiçào! Assim. z atinge máximo.

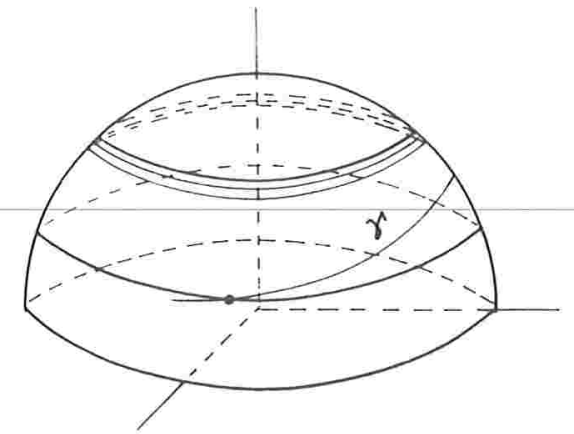

Figura 10a

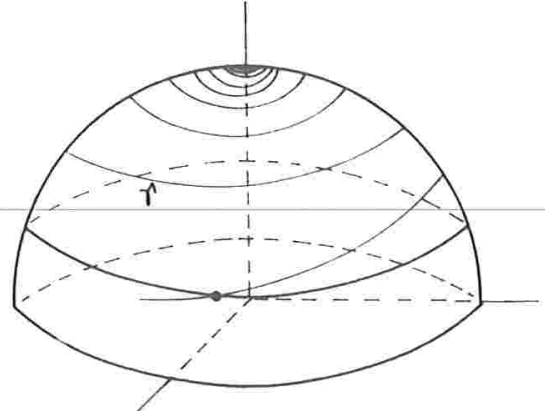

Figura 10b

Por reflexào segundo um conveniente meridiano, ortogonal ao bordo, a curva ? intercepta novamente o paralelo $z \equiv z_{a}$, digamos num $\theta_{1}$. Suponhamos ainda que $z$ seja monótona decrescente para $\theta>\theta_{1}$. Entào, ou a curva $\gamma$ espirala em torno de um paralelo $z \equiv z_{2}, 0<z_{2}<z_{a}$ (veja Figura 11a) ou, espirala em torno do bordo de $S_{+}^{2}$ (veja Figura 11b).

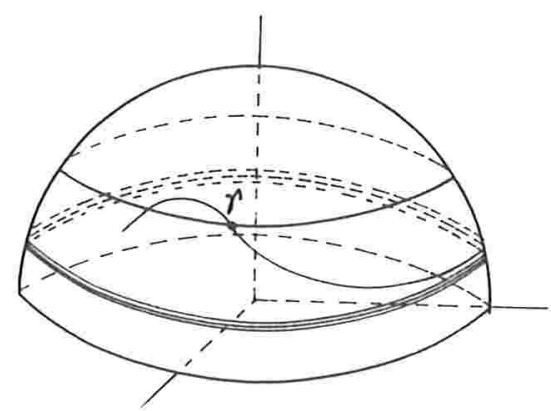

Figura 11a

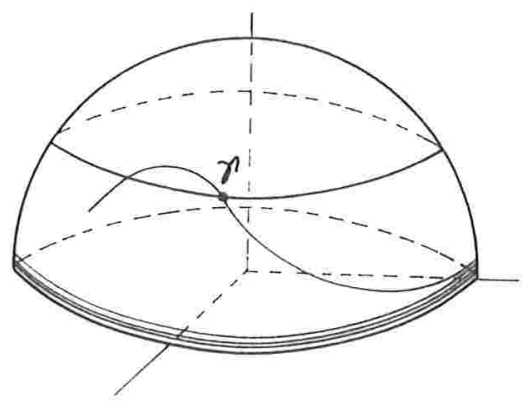

Figura 11b

Se ocorresse a primeira situação teríamos uma contradição (análogo ao caso da Figura 
10a). Se ocorresse o segundo caso. existiria um ponto $(\bar{\theta}, \bar{z})$ na curva onde $\lambda_{1}=k g \approx 0$ e $\dot{z}(\bar{\theta}) \approx 0$. Agora, nesse ponto. teríamos $\dot{\theta} \approx 1$ e $\bar{z} \approx 0$. Vejamos que em valor absoluto $\lambda_{2}$ é muito grande. Para isto, consideremos o volume $r$ da órbita passando por esse ponto. Entào

$$
\begin{aligned}
\left|\lambda_{2}\right| & =\left|\frac{\partial}{\partial \mathbf{n}}(\ln v)\right| \\
& =\left|\frac{\partial}{\partial \mathbf{n}}(\ln (2 \pi \bar{z}))\right| \\
& =\left|\left\langle 0 \mathbf{e}_{\theta}-\frac{\sqrt{1-\bar{z}^{2}}}{\bar{z}} \mathbf{e}_{\bar{z}}, \frac{\dot{\bar{z}}}{\sqrt{1-\bar{z}^{2}}} \mathbf{e}_{\theta}-\dot{\theta} \sqrt{1-\bar{z}^{2}} \mathbf{e}_{\bar{z}}\right\rangle\right| \\
& =\frac{\dot{\theta} \sqrt{1-\bar{z}^{2}}}{\bar{z}} .
\end{aligned}
$$

Olhando para o último quociente temos que $\left|\lambda_{2}\right|$ é um número grande. Vejamos que isto nos leva a uma contradiçào. Lembremos da equaçào 2.2 que

$$
\ell\left(\frac{\left(\lambda_{1}-\lambda_{2}\right)^{2}}{4}\right)=\frac{\left(\lambda_{1}-\lambda_{2}\right)^{2}}{4 \lambda_{1}{ }^{2} \lambda_{2}{ }^{2}} .
$$

Assim, por um lado

$$
,\left(\frac{\left(\lambda_{1}-\lambda_{2}\right)^{2}}{4}\right) \approx \frac{\lambda_{2}{ }^{2}}{4 \lambda_{1}{ }^{2} \lambda_{2}{ }^{2}}=\frac{1}{4 \lambda_{1}{ }^{2}}
$$

que, portanto é muito grande. Por outro lado,

$$
\frac{\left(\lambda_{1}-\lambda_{2}\right)^{2}}{4} \approx \frac{\lambda_{2}^{2}}{4}
$$

que também é muito grande. Mas $\iota(x) \longrightarrow 0$ quando $x \longrightarrow+\infty$ ! Logo chegamos numa contradição. Desta forma $z=z(\theta)$ possui um mínimo, como queríamos demonstrar.

Finalmente, façamos um estudo sobre os períodos das curvas soluçòes que interceptam o paralelo $z \equiv z_{a}=\sqrt{\frac{1}{2}+\frac{1}{2} \sqrt{1-a}}$, onde $a=\frac{1}{\bar{x}} \operatorname{com} \bar{x}$ tal que $\iota(\bar{x})=\bar{x}$. 
Já vimos que qualquer dessas tais curvas soluçòes sào periódicas. Se i é uma dessas soluçoes. e $\gamma$ atinge dois pontos críticos consecutivos em $\tilde{z}_{1}=z_{(}\left(\theta_{1}\right)$ e $z_{2}=z_{(}\left(\theta_{2}\right)$. o seu período, $\Theta$, em relaçào a $\theta$. é dado por $2\left|\theta_{1}-\theta_{2}\right|$.

Estamos interessados na existência de curvas soluçòes que sào fechadas.

Lema 2.2.3 Sejam $\theta_{1} \in \theta_{2}$ dois pontos críticos consecutivos de $z=z(\theta)$. Se $\theta_{1}-\theta_{2}= \pm r \pi, r$ um número racional nâo negativo, entâo a curva soluçào $\gamma=(\theta . z)$ é fechada com período $2 r \pi . e m$ relaçào a $\theta$. em particular. se $\quad r=\frac{1}{n}, \quad n \in \mathbb{N}$ então y é uma curva fechada simples com período $\frac{2 \pi}{n}$.

Demonstração: Se $\theta_{1}$ é um ponto crítico de $z=z(\theta)$. O Lema 2.2.1 nos diz que $\gamma$ é invariante pela reflexào em relaçào ao meridiano $\theta=\theta_{1}$. O mesmo ocorrendo para $\theta_{2}$. Pelo Lema 2.2.2, $\gamma$ é periódica e o período é $=2 \pi r, \quad r$ um número racional positivo. Desde que os máximos e mínimos ocorrem sempre em $z_{1}$ e $z_{2}$ (ou vice-versa), a curva fecha. No caso particular em que $r=\frac{1}{n}, \quad n \in \mathbb{N}$, entào a curva fecha na primeira volta, ou seja, é fechada simples.

Agora, por dependência contínua das curvas soluções de uma EDO de segunda ordem com relação às condições iniciais, se fixarmos $\theta_{0}$ e $z\left(\theta_{0}\right)=z_{a}$, entào soluçôes cujas condições iniciais $\dot{z}\left(\theta_{0}\right)$ "próximas" serão "próximas", de modo que seus períodos serão "próximos" também. Desta forma vemos que os períodos das soluçòes que cortam o paralelo $z=z_{a}$ em $\left(\theta_{0}, z_{a}\right)$ dependem continuamente da variação de $\dot{z}\left(\theta_{0}\right)=\dot{z}_{0}$. Como temos os meridianos como soluções, por dependência contínua existem soluções "muito próximas" destes e, portanto, com períodos "muito próximos" de $\pi$. Devemos nos lembrar que tais curvas "acompanham " os correspondentes meridianos arbitrariamente "próximas" destes, e portanto, para cada uma delas, seus pontos de mínimos ocorrerào 
muito "próximos" dos pontos de intersecçào de um meridiano com o bordo de $S_{+}^{2}$. Portanto existe uma seqüência infinit a de números racionais $r_{i}$. arbitrariamente próximos de $\pi$. tais que cada $r_{i} \pi$ é período de uma curva soluçào. Isto acaba de estabelecer o Teorema A. 


\section{Capítulo 3}

\section{Sobre certas superfícies rotacionais}

\section{especiais de tipo mínimo em $S^{3}$}

Dedicaremos este capítulo ao estudo das superfícies rotacionais especiais de tipo mínimo em $S^{3}$ do tipo $f(x)=c \sqrt{x}, 0<c<1$.

\subsection{Sobre a EDO satisfeita por uma curva geradora}

Começamos apresentando a EDO que deve ser satisfeita pela curva geradora de uma tal superfície.

Proposição 3.1.1 Seja $\gamma=(\theta, z)$ uma curva $C^{\infty} \mathrm{em} S_{+}^{2}$, parametrizada pelo comprimento de arco $s$. Então a correspondente superfície $M=\pi^{-1}(\gamma) \subset S^{3}$ é uma superfície especial rotacional de tipo mínimo se, e somente se, uma das seguintes equaçôes diferenciais é satisfeita:

$$
z \ddot{z}+z^{2}=\left(\frac{1+c}{1-c}\right)\left(1-z^{2}-\dot{z}^{2}\right) \quad \text { se } \dot{\theta} \geq 0
$$




$$
z \ddot{z}+z^{2}=\left(\frac{1-c}{1+c}\right)\left(1-z^{2}-\dot{z}^{2}\right) \quad \text { se } \dot{\theta} \leq 0 .
$$

onde $c$ é dada por $f(x)=c \sqrt{x}, 0<c<1$.

Demonstração: Seja $f$ dada por $f(x)=c \sqrt{x}, 0<c<1$. Assim, a equaçào dada em 1.12) da Proposiçào 1.2.2 é equivalente à seguinte equaçào:

$$
z \ddot{z}+z^{2}-\left(1-z^{2}-\dot{z}^{2}\right)= \pm 2 c z \sqrt{1-z^{2}-\dot{z}^{2}} \sqrt{\frac{\left(z \ddot{z}+z^{2}+\left(1-z^{2}-\dot{z}^{2}\right)\right)^{2}}{4 z^{2}\left(1-z^{2}-\dot{z}^{2}\right)}},
$$

onde + ou - depende do mesmo sinal de $\dot{\theta}$. Agora, desde que $K=\lambda_{1} \lambda_{2}<0$, as expressòes de $\lambda_{1}$ e $\lambda_{2}$ em 1.11 do Capítulo 1 nos dào que $\lambda_{1}>0$ e $\lambda_{2}<0$ se $\dot{\theta}>0$, de modo que $0<\lambda_{1}-\lambda_{2}=\frac{\ddot{z} z+z^{2}+\left(1-z^{2}-\dot{z}^{2}\right)}{z \sqrt{1-z^{2}-\dot{z}^{2}}}$. Analogamente, se $\dot{\theta}<0$, entào $\lambda_{1}<0$ c $\lambda_{2}>0$ e, conseqüentemente, $0<\lambda_{2}-\lambda_{1}=\frac{\ddot{z} z+z^{2}+\left(1-z^{2}-\dot{z}^{2}\right)}{z \sqrt{1-z^{2}-\dot{z}^{2}}}$. Assim, a equaçào dada em (3.2), acima, nos leva às equaçòes 3.1 a e 3.1 b desejadas.

Cada uma das equaçôes da Proposiçào 3.1.1 podem ser reduzidas a uma equação de primeira ordem. Estabelecemos este resultado no seguinte lema que será útil na análise do comportamento das curvas geradoras das superfícies especiais de tipo mínimo em questão.

Lema 3.1.1 As equaçôes 3.1a e $3.1 b$ na Proposição 3.1.1 podem ser reduzidas à equaçào diferencial de primeira ordem

$$
\begin{aligned}
& \dot{z}^{2}=1-z^{2}-\frac{a}{z^{2 \alpha}}, \\
& \text { onde a é uma constante positiva e } \alpha=\left\{\begin{array}{l}
\frac{1+c}{1-c} \text { no caso } \dot{\theta} \geq 0 \\
\frac{1-c}{1+c} \quad \text { no caso } \dot{\theta} \leq 0 .
\end{array}\right. \text {. }
\end{aligned}
$$

Demonstração: Multiplicando a equaçào 3.1a por $\underset{z}{\frac{\dot{z}}{z}}$ obtemos:

$$
\frac{(\ddot{z}+z) \dot{z}}{1-\tilde{z}^{2}-\dot{z}^{2}}=a \frac{\dot{z}}{z} \text {. }
$$


Integrando esta, temos que

$$
1-z^{2}-\dot{z}^{2}=a z^{-20} . \quad a=\text { constante. } a>0,
$$

de onde segue o resultado para o caso $\dot{\theta} \geq 0$. A demonstraçào do caso $\dot{\theta} \leq 0$ é completamente análoga.

Observação 3.1.1 (a) As únicas soluçòes de (3.1a) passando por $o=(0,0,1,0) \in S_{+}^{2}$ são do tipo $\theta=$ constante, ou seja, sào os círculos geodésicos ortogonais ao bordo de $S_{+}^{2}$ (Figura 12b). As superfícies rotacionais correspondentes sào as 2-esferas totalmente geodésicas de $S^{3}$.

(b) Para cada $a \in \mathbb{R}, 0<\alpha<1$, a única soluçào tal que $\dot{z} \equiv 0$ é dada por $z \equiv \sqrt{\frac{a}{a+1}}$ (Figura 12a). De fato, $\ddot{z} \equiv 0$ e (3.1a) se resolve para $z$ nos dando o resultado acima. A superfície rotacional correspondente em cada classe de superfícies especiais de tipo mínimo, dada por a nas condiçôes acima, é o toro flat

$$
S^{1}\left(\sqrt{\frac{\alpha}{\alpha+1}}\right) \times S^{1}\left(\sqrt{\frac{1}{\alpha+1}}\right) .
$$

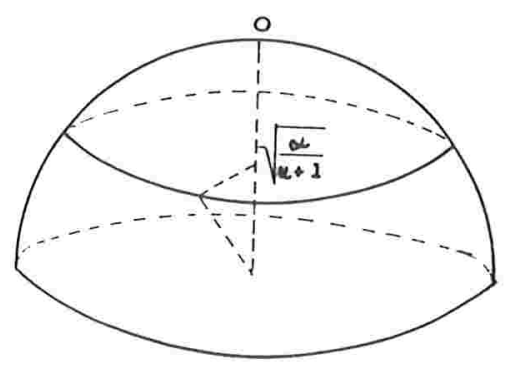

Figura 12a

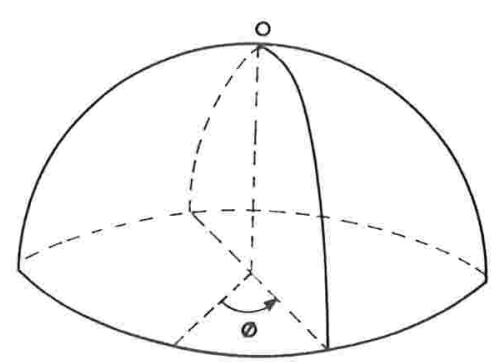

Figura $12 b$

(c) Do mesmo modo como vimos anteriormente, pelo Teorema de Existência e Unicidade de soluçòes de uma EDO de segunda ordem, existem soluçòes locais da equaçào 3.1a, 
uma para cada constante positiva a dada por (3.3) do Lema 3.1.1, ou seja, uma para cada $a=z_{0}^{2 a}\left(1-z_{0}^{2}-\dot{z}_{0}^{2}\right)$. onde $z_{0}=z\left(s_{0}\right)$ e $\dot{z}\left(s_{0}\right)=\dot{z}_{0}$. Por continuação analítica estas soluçòes podem ser estendidas para todos os valores de $s \in \mathbb{R}$. Por ser parametrizada pelo comprimento de arco, cada curva soluçào nào pode permanecer constante em qualquer subintervalo de $\mathbb{R}$. Além disso, por unicidade, nenhuma outra curva-soluçào é tangente a qualquer "meridiano" ortogonal ao bordo de $S_{+}^{2}$ e nenhuma tal curva toca o bordo, a nào ser ortogonalmente.

(d) Podemos supor daqui por diante que se a curva solução de (3.1a) (respectivamente (3.1b)) nào é um grande círculo, entào $\dot{\theta}>0$ (respectivamente, $\dot{\theta}<0$ ) sempre. Escolheremos uma orientaçào de $\gamma$ de modo a sempre trabalharmos com o caso $\dot{\theta}>0$. Assim todas as curvas soluçòes podem ser expressas como $z=z(\theta)$ onde $\theta$ percorre todos os números reais.

\subsection{Sobre o problema do mergulho}

Após demonstrarmos que a curva geradora de uma superfície de tipo mínimo,em qualquer das classes em questão, é periódica faremos uma estimativa de um tal período. e isto nos permitirá demonstrar o seguinte resultado:

\section{Teorema B}

Em cada classe de superfícies especiais de tipo mínimo determinada por $f$ do tipo $f(x)=c \sqrt{x}, c \in \mathbb{R}, c<0<1$, a única superfície especial compacta, homeomorfa a $S^{1} \times S^{1}$ e mergulhada em $S^{3}$ é o toro flat $S^{1}\left(\sqrt{\frac{a}{a+1}}\right) \times S^{1}\left(\sqrt{\frac{1}{a+1}}\right)$. onde $a=\frac{1+c}{1-c}$. 
Comecemos estudando a periodicidade da curva geradora de uma superfície especial rotacional de tipo mínimo nas classes particulares em questào. No que segue. M nào será uma 2-esfera totalmente geodésica.

Daqui por diante, a denotará o número real $\frac{1+c}{1-c}, 0<c<1$ (veja enunciado do Teorema B).

Proposição 3.2.1 Seja $M \hookrightarrow S^{3}$ uma superficie especial completa rotacional de tipo mínimo na classe de uma função $f$ do tipo $f(x)=c \sqrt{x}, 0<c<1$. Então a curva geradora, $\gamma=\pi(M)$, de $M$ é uma curva periódica em $S_{+}^{2}$.

Demonstração: Fixemos uma das tais classes de superfícies de tipo mínimo, ou seja, fixemos $\alpha, \alpha>1$. A curva $\gamma=(\theta(s), z(s))$, parametrizada pelo comprimento de arco, é uma curva soluçào da equaçào diferencial ordinária (3.1a). Suponhamos, sem perda de generalidade, que $\gamma$ é uma curva tal que $\dot{\theta}(s)>0$ sempre. Se $\gamma$ é a curva soluçào correspondente a um toro flat (veja Observação 3.1.1b) o resultado é obviamente verdadeiro. Suponhamos então que $\dot{z}$ não seja identicamente nulo. Afirmamos que $z$ atinge mínimo e máximo. Consideremos os três casos abaixo:
a) $z\left(s_{0}\right)=\sqrt{\frac{\alpha}{\alpha+1}}$,
b) $z\left(s_{0}\right)>\sqrt{\frac{a}{\alpha+1}}$,
c) $z\left(s_{0}\right)<\sqrt{\frac{\alpha}{\alpha+1}}$.

Caso a) Este caso decorre imediatamente do Lema 2.2.2 do Capítulo 2, pois aquele resultado foi obtido para qualquer classe de superfícies de tipo mínimo.

Caso b) Se $z\left(s_{0}\right)>\sqrt{\frac{a}{a+1}}$, suponhamos novamente que $z$ não atinge um mínimo. Assim, suponhamos que $\dot{z}(s)<0$ se $s>s_{0}$. Teríamos entào que $\lim _{s \rightarrow+\infty} z(s)=0$ ou $\lim _{s \rightarrow+\infty} z(s)=z_{0}$, onde $0<z_{0}<\sqrt{\frac{a}{a+1}}$ ou $\sqrt{\frac{a}{a+1}}<z_{0}<z\left(s_{0}\right)$. No primeiro caso a curva estaria espiralando 
em torno do bordo de $S_{+}^{2}$ e, no segundo, a curva estaria espiralando em torno do paralelo $z \equiv z_{0}$ (veja Figura 13a). Novamente. por argumentaçòes utilizadas no Lema 2.2.2. seríamos levados a conclusòes contraditórias. Restaria entào a possibilidade de $\gamma$ espiralar em torno da curva soluçào $z \equiv \sqrt{\frac{a}{a+1}}($ veja Figura 13b). Assim, pelo caso a). existiria uma curva soluçào $\bar{\gamma}=(\bar{\theta}, \bar{z})$, cortando o paralelo acima $\operatorname{com} \bar{\gamma}\left(s_{1}\right)=\gamma\left(s_{1}\right)$ e $\dot{\bar{z}}\left(s_{1}\right)=\dot{z}\left(s_{1}\right)$, para algum $s_{1}>s_{0}$. De fato, por dependência contínua das soluçôes de uma EDO de segunda ordem, com relaçào às condiçòes iniciais, existiria uma curva soluçào $\bar{\gamma}$, interceptando o paralelo $z \equiv \sqrt{\frac{a}{a+1}}$ e " muito próxima" do mesmo. Concluiríamos, pelo teorema de existência e unicidade para uma EDO de segunda ordem, que $\bar{\gamma} \equiv \gamma$ (contradiçào!). Assim, z atinge um mínimo. Para mostrarmos, neste caso, que z atinge um máximo, os argumentos sào os mesmos que os já utilizados no Lema 2.2.2.

Caso c) Seja $z\left(s_{0}\right)<\sqrt{\frac{\alpha}{\alpha+1}}$. Afirmamos que $z$ atinge mínimo (a demonstraçào é a mesma que para a situaçào correspondente do caso a)). Também, temos que $z$ atinge um máximo. A demonstraçào usa argumentaçòes semelhantes às já utilizadas em situaçòes correspondentes dos casos a) e b).

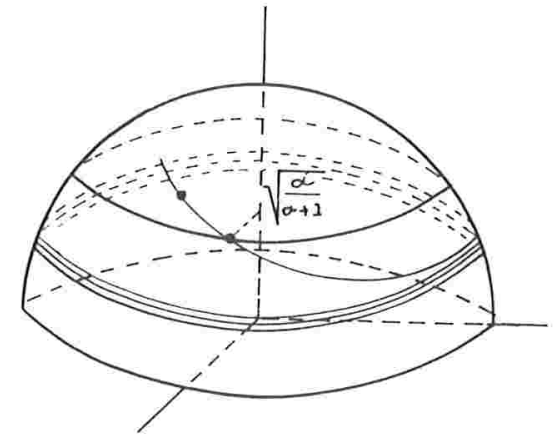

Figura 13a

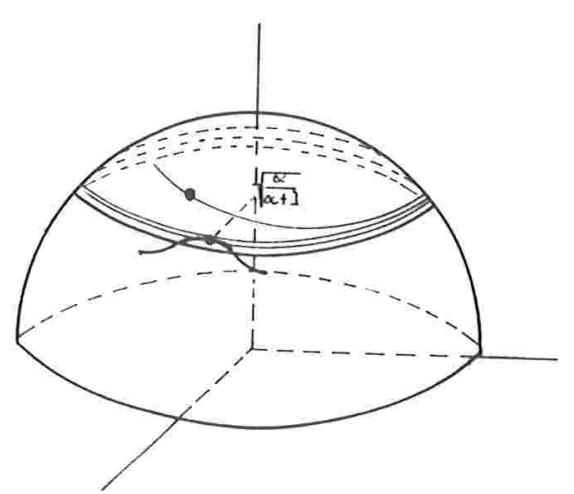

Figura 13b

Logo, nos três casos considerados, $\gamma$ possui, pelo menos, dois pontos críticos e, pelo Corolário 2.2.1, temos que a curva y é periódica. 
É importante que observemos ainda alguns fatos sobre cada curva soluçào $\uparrow$.

Observação 3.2.1 (a) Fixemos uma classe de superfícies especiais de tipo mínimo em questão, digamos uma classe dada por $f(x)=c \sqrt{x}, 0<c<1$. Seja $\alpha=\frac{1+c}{1-c}$. Vimos na Observaçào 3.1 .1 b que o toro flat $S^{1}\left(\sqrt{\frac{a}{\alpha+1}}\right) \times S^{1}\left(\sqrt{\frac{1}{\alpha+1}}\right)$ é uma superfície nesta classe e corresponde ao paralelo dado por $z \equiv \sqrt{\frac{\alpha}{\alpha+1}}$ em $S_{+}^{2}$. Uma observação que fazemos agora é a de que cada curva geradora $\gamma$ de uma superfície rotacional nesta classe "corta" a curva $z \equiv \sqrt{\frac{a}{\alpha+1}}$ do toro flat. Em outras palavras, se $\gamma$ atinge um mínimo num ponto $s_{1} \in \mathbb{R}$ entào $z\left(s_{1}\right)<\sqrt{\frac{\alpha}{\alpha+1}}$ e se $\gamma\left(s_{2}\right)$ é um máximo entào $z\left(s_{2}\right)>\sqrt{\frac{\alpha}{\alpha+1}}$. De fato, se $s_{1}$ é um ponto de mínimo, entào $\ddot{z}\left(s_{1}\right)>0$ e $\dot{z}\left(s_{1}\right)=0$. Assim, utilizando a equaçào 3.1a temos que

$$
a=\frac{z\left(s_{1}\right) \ddot{z}\left(s_{1}\right)+z^{2}\left(s_{1}\right)}{1-z^{2}\left(s_{1}\right)}>\frac{z^{2}\left(s_{1}\right)}{1-z^{2}\left(s_{1}\right)},
$$

de onde $z\left(s_{1}\right)<\sqrt{\frac{\alpha}{\alpha+1}}$. Analogamente, para o máximo.

(b) Em decorrência da observação a) acima, cada solução de (3.1a) passa por $z(0)=$ $\sqrt{\frac{\alpha}{\alpha+1}}($ lembremos que a classe das superfícies especiais está fixada e que por simplificação tomamos $\left.s_{0}=0\right)$. Assim, cada soluçâo $\operatorname{com} \dot{z}(0)$ tal que $z^{2}(0)+\dot{z}^{2}(0) \leq 1$, ou $0 \leq \dot{z}^{2}(0) \leq$ $\frac{1}{\alpha+1}$, corresponde uma solução de $3.2 \operatorname{com} 0<a \leq\left(\frac{\alpha}{\alpha+1}\right)^{\alpha}\left(\frac{1}{\alpha+1}\right)$ e vice-versa.

(c) Continuando com $\alpha$ fixo, não é difícil ver que, impondo $\dot{z}=0$, a equação

$$
z^{2 \alpha}\left(1-z^{2}\right)=a
$$

onde $0<a<\left(\frac{\alpha}{\alpha+1}\right)^{\alpha}\left(\frac{1}{\alpha+1}\right)$, possui exatamente duas raízes reais $z_{1}$ e $z_{2} \mathrm{com}$

$$
0<z_{1}<\sqrt{\frac{a}{\alpha+1}}<z_{2}<1
$$

Assim, o mínimo e o máximo de $z$ devem ser, respectivamente, $z_{1}$ e $z_{2}$, de modo que $z_{1}<z(s)<z_{2}$ para todo $s \in \mathbb{R}$. 
Como $\dot{\theta}>0$ sempre. cada curva soluçào ; pode ser dada na forma $z=z(\theta)$. E mais. segundo o Lema 2.2.1. a curva ; é simétrica em relaçào a $\theta=\theta_{1}$ (respectivamente $\theta_{2}$ ). onde $z_{1}=z\left(\theta_{1}\right)$ (respectivamente $z_{2}=z\left(\theta_{2}\right)$ ) é o mínimo de $z$ (respectivamente. o máximo de $\approx$ ).

A Proposiçào 3.2.1 nos diz que a curva soluçào. $\gamma$. passando por $z(0)=\sqrt{\frac{a}{\alpha+1}}$ (a fixo). correspondente a um valor de $a .0<a \leq\left(\frac{a}{a+1}\right)^{\alpha}\left(\frac{1}{a+1}\right)$, é periódica. O seu período, $\Theta(a)$. em relação a $\theta$, é dado por $2\left|\theta_{1}-\theta_{2}\right|$.

Para estudarmos o problema do mergulho de superfícies "tóricas", de gênero um, em $S^{3}$, devemos analisar se a curva solução fecha, ou nào, na primeira volta.

Lembremos que para cada $a \in \mathbb{R}, \alpha>1$, a classe de superfícies especiais de tipo mínimo correspondente possui um único toro flat, a saber. $S^{1}\left(\sqrt{\frac{a}{\alpha+1}}\right) \times S^{1}\left(\sqrt{\frac{1}{\alpha+1}}\right)$ mergulhado em $S^{3}$ (veja Observaçào $3 \cdot 1 \cdot 1 \mathrm{~b}$ ).

Resta-nos então apresentar estimativas dos períodos das curvas geradoras das superfícies em questão, que nos levem às conclusões desejadas.

Com este propósito, observemos que uma superfície rotacional $M$, imersa em $S^{3}$, em questão, pode ser vista como o lugar de se mover círculos em subespaços afins paralelos a $\{(0,0)\} \times \mathbb{R}^{2}$, ao longo de uma curva plana, em $\mathbb{R}^{2} \times\{(0,0)\}$, descrita pelos seus centros. De fato, pela descriçào da geometria orbital da açào de $G$ sobre $S^{3}$, apresentada na Seçào 1.2 do Capítulo 1, cada tal círculo é uma $G$-órbita de um ponto $\gamma(s) \in S_{+}^{2} \subset S^{3}$, onde $\gamma=M / G$. Assim, a curva plana, $\beta(s)$, ao longo da qual movemos os círculos, é obtida projetando-se ortogonalmente a curva $\gamma$ em $S_{+}^{2}$ sobre o disco unitário cujo bordo coincide $\operatorname{com} \partial S_{+}^{2}$.

Se $q \in \mathbb{R}^{2} \times\{(0,0)\}$ é um ponto genérico da curva $\beta$, podemos definí-lo a partir da funçào suporte $h(\phi)$ de $\beta$ (veja Figura 14) por

$$
q(\phi)=e^{i(\phi-\pi / 2)}(h(\phi+i \dot{h}(\phi))
$$


identificando $\mathbb{R}^{2}$ com o plano complexo.

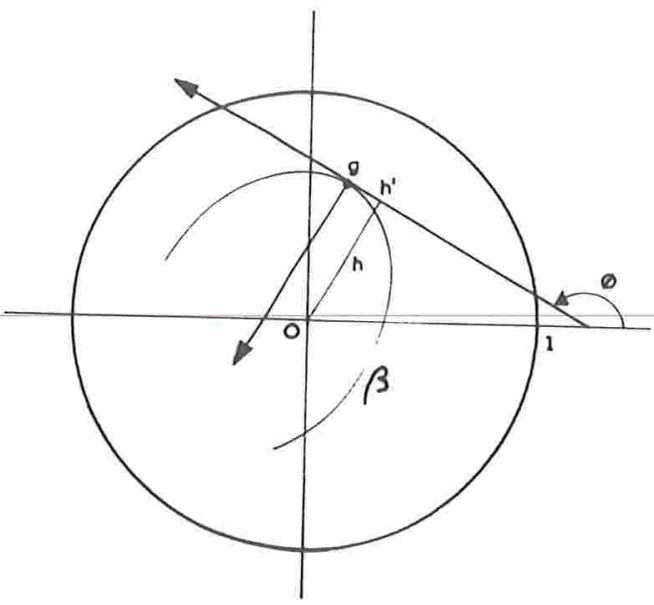

Figura 14

Em [O1, pp. 158 e 159], T. Otsuki obteve as seguintes expressòes para as curvaturas principais de uma tal superfície $M \hookrightarrow S^{3}$ :

$$
\left\{\begin{array}{l}
\lambda_{1}=k g=-\frac{h}{\sqrt{1-h^{2}}}+\frac{1-h^{2}-h^{2}}{(h+\ddot{h}) \sqrt{\left(1-h^{2}\right)^{3}}} \\
\lambda_{2}=-\frac{h}{\sqrt{1-h^{2}}} .
\end{array}\right.
$$

Escolhemos a orientaçào de $\gamma$ tal que $\lambda_{1}>0$ e $\lambda_{2}<0$. Aqui $\dot{h}=\frac{\mathrm{d} h}{\mathrm{~d} \phi}$ e $\ddot{h}=\frac{\mathrm{d}^{2} h}{\mathrm{~d} \phi^{2}}$ e lembremos que a condiçào de $M$ ser uma superfície especial de tipo mínimo na classe de uma fixada $f$ dada por $f(x)=c \sqrt{x}, 0<c<1$ é a seguinte (veja Observação 1.1.3b):

$$
(1-c) \lambda_{1}+(1+c) \lambda_{2}=0 .
$$

Substituindo as expressôes de $\lambda_{1}$ e $\lambda_{2}$, dadas acima obtemos:

$$
(1-c)\left(-\frac{h}{\sqrt{1-h^{2}}}+\frac{1-h^{2}-\dot{h}^{2}}{(h+\ddot{h}) \sqrt{\left(1-h^{2}\right)^{3}}}\right)-(1+c) \frac{h}{\sqrt{1-h^{2}}}=0
$$

que, por sua vez, equivale a

$$
\frac{2}{1-c}\left[h(h+\ddot{h})\left(1-h^{2}\right)\right]-\left(1-h^{2}-\dot{h}^{2}\right)=0 .
$$


a qual se reescreve como

$$
(a+1) h\left(1-h^{2}\right) \ddot{h}+\dot{h}^{2}+\left(1-h^{2}\right)\left((a+1) h^{2}-1\right)=0 .
$$

Vemos assim que qualquer curva soluçào da equaçào 3.1a se projeta sobre uma curva soluçào da equaçào 3.9 .

Fazendo $u=h^{2}-\dot{h}^{2}$, obtemos

$$
\begin{aligned}
\frac{1}{2} \frac{\mathrm{d} u}{\mathrm{~d} \phi} & =h \dot{h}+\dot{h} \ddot{h} \\
& =h \dot{h}-\frac{\dot{h}^{3}}{(\alpha+1) h\left(1-h^{2}\right)}-\frac{\left((\alpha+1) h^{2}-1\right) \dot{h}}{(\alpha+1) h},
\end{aligned}
$$

ou seja,

$$
\frac{1}{2} \frac{\mathrm{d} u}{\mathrm{~d} \phi}=\frac{\dot{h}\left(1-h^{2}-\dot{h}^{2}\right)}{(\alpha+1) h\left(1-h^{2}\right)}=\frac{\dot{h}(1-u)}{(\alpha+1) h\left(1-h^{2}\right)},
$$

de modo que

$$
\frac{\mathrm{d} u}{1-u}=\frac{2}{\alpha+1}\left(\frac{1}{h}+\frac{1}{2(1-h)}-\frac{1}{2(1+h)}\right) \mathrm{d} h
$$

Integrando esta equação, obtemos

$$
1-u=C\left(\frac{h^{2}}{1-h^{2}}\right)^{-\frac{1}{\alpha+1}}, C=\text { constante }
$$

da qual obtemos

$$
\left(\frac{\mathrm{d} h}{\mathrm{~d} \phi}\right)^{2}=1-h^{2}-C\left(\frac{1}{h^{2}}-1\right)^{\frac{1}{\alpha+1}} .
$$

Observação 3.2.2 (a) Fixado $\alpha=\frac{1+c}{1-c},(\alpha>1)$, a única soluçào constante da equação 3.9. dada por $h(\phi)=\sqrt{\frac{1}{\alpha+1}}$ corresponde ao toro flat $S^{1}\left(\sqrt{\frac{\alpha}{\alpha+1}}\right) \times S^{1}\left(\sqrt{\frac{1}{\alpha+1}}\right)$.

(b) Impondo que $\dot{h}=0$ na equação 3.11 obtemos a seguinte equação

$$
1-h^{2}-C\left(\frac{1}{h^{2}}-1\right)^{\frac{1}{\alpha+1}}=0 .
$$


onde, fazendo $h^{2}=x$, obtemos

$$
1-x-C\left(\frac{1}{x}-1\right)^{\frac{1}{a+1}}=0 .
$$

As raízes da equaçào acima sào obtidas pelas possíveis intersecçòes das curvas $y=$ $\left(\frac{1}{x}-1\right)^{\frac{1}{\alpha+1}}(0<x<1)$ e da reta $y=\frac{1}{C}(1-x)$. Nào é difícil ver que a curva e a reta, dadas acima, se interceptam em exatamente dois pontos, quando

$$
0<C<\left(\frac{\alpha}{\alpha+1}\right)^{\frac{\alpha}{\alpha+1}}\left(\frac{1}{\alpha+1}\right)^{\frac{1}{\alpha+1}}
$$

e se tangenciam no ponto $\left(\frac{1}{\alpha+1}, \alpha^{\frac{1}{\alpha+1}}\right)$ quando $C=\left(\frac{\alpha}{\alpha+1}\right)^{\frac{\alpha}{\alpha+1}}\left(\frac{1}{\alpha+1}\right)^{\frac{1}{\alpha+1}}$. Desta forma, a equação 3.12 possui exatamente duas raízes, digamos $h_{1}$ e $h_{2}$ com

$$
0<h_{1}<\sqrt{\frac{1}{\alpha+1}}<h_{2}<1
$$

Os valores $h_{1}$ e $h_{2}$ sào, respectivamente, o mínimo e o máximo de $h(\phi)$.

(c) A soluçào $\phi \equiv$ constante da equação 3.9 corresponde à solução $\theta \equiv$ constante da equação 3.1a, a qual é a curva geradora de uma 2-esfera totalmente geodésica em $S^{3}$.

(d) Se $\phi$ não é constante, então a solução $h$ da equaçào 3.9 é dada por $h=h(\phi)$. E mais, $h=h(\phi)$ é simétrica em relação a $\phi=\phi_{1}$ ou $\phi=\phi_{2}$, onde $h_{1}=h\left(\phi_{1}\right)$ e $h_{2}=h\left(\phi_{2}\right)$. Logo, da equaçào 3.11 , temos que $h(\phi)$ é periódica e seu período, $\Theta(C)$, é dado por

$$
\Theta(C)=2 \int_{h_{1}}^{h_{2}} \frac{\mathrm{d} h}{\sqrt{1-h^{2}-C\left(\frac{1}{h^{2}}-1\right)^{\frac{1}{\alpha+1}}}}, \quad 0<C<\left(\frac{\alpha}{\alpha+1}\right)^{\frac{\alpha}{\alpha+1}}\left(\frac{1}{\alpha+1}\right)^{\frac{1}{\alpha+1}} .
$$

É importante observarmos que as soluções $h(\phi)$ da equação 3.9 e $z(\theta)$ da equação 3.1 a são as mesmas. Portanto, estimaremos o período $\Theta(C)$.

Lema 3.2.1 (Otsuki [O1]) Para cada $a>1$, o período $\Theta(C)$ acima definido tem as seguintes propriedades: 
(i) é diferenciável sobre $0<C<\left(\frac{a}{\alpha+1}\right)^{\frac{\alpha}{\alpha+1}}\left(\frac{1}{\alpha+1}\right)^{\frac{1}{\alpha+1}}$ :

(ii) $\Theta(C)>\pi$ :

(iii) $\lim _{C \rightarrow 0^{+}} \Theta\left(C^{\prime}\right)=\pi$ :

(iv) $\lim _{C^{\prime} \rightarrow b} \Theta(C)=\sqrt{2} \pi$. onde $b=\left(\frac{\alpha}{a+1}\right)^{\frac{a}{a+1}}\left(\frac{1}{1+a}\right)^{\frac{1}{1+\alpha}}$

Demonstração: As demonstraçòes de (i) - (iv) seguem as demonstrações dos Lemas 1, 2,3 e 5 em [O1]. Observemos que nos lemas citados temos " $n$ " inteiro positivo no lugar de " $\alpha+1$ ", mas suas demonstraçòes permanecem válidas quando colocamos " $\alpha+1$ " no lugar de "n".

Lema 3.2.2 (Otsuki, [O2]) Para cada $a>1, \Theta(C)$ satisfaz

$$
\Theta(C)<\left(\frac{1}{\sqrt{2}}+\sqrt{\frac{\alpha}{\alpha+1}}\right) \pi<\sqrt{2} \pi .
$$

Demonstração: Também segue a risca a demonstração do teorema obtido em [O2], por Otsuki.

\section{Demonstração do Teorema B}

Para cada $\alpha>1$ e para cada $C, 0<C<\left(\frac{\alpha}{\alpha+1}\right)^{\frac{a}{\alpha+1}}\left(\frac{1}{\alpha+1}\right)^{\frac{1}{\alpha+1}} ; \Theta(C)=\frac{2 \pi}{n},(n \in \mathbb{N})$, não ocorre entre $\pi$ e $\sqrt{2} \pi$. Portanto, a correspondente superfície compacta, homeomorfa a $S^{1} \times S^{1}$, possui auto-intersecções, não sendo mergulhada. Concluimos então que a única superfície de tipo mínimo, compacta de gênero um e mergulhada em $S^{3}$, na classe de uma $f$ do tipo $f(x)=c \sqrt{x}, 0<c<1$, é o toro flat $S^{1}\left(\sqrt{\frac{\alpha}{\alpha+1}}\right) \times S^{1}\left(\sqrt{\frac{1}{\alpha+1}}\right)$ na classe. 


\section{Referências}

[BdCH] Back, A.: do Carmo. M.P. \& Hsiang. W.Y. On some fundamental equations of equivariant Riemannian geometry. Preprint.

[BN] Brauer, F. \& Nohel, J.A. Qualitative theory of ordinary differential equations. W.A. Benjamin, Inc., New York, 1969.

[BL] Brito, F.G.B. \& Leite, M.L. A remark on rotational hypersurfaces of $S^{n}$. Bull. Soc. Math. Belg., Ser. B, 42 (1990), 303-318.

[dC] do Carmo, M.P. Geometria Riemanniana. Projeto Euclides, IMPA/CNPq, Rio de Janeiro, 1979.

[dCD] do Carmo, M.P. \& Djaczer, M. Rotational hypersurfaces in spaces of constant curvature. Transactions of the A.M.S., 277 (1983), 685-709.

[Ch] Charlet, B. Le problème de Bernstein sphérique. Sem. Palaiseau, Asterisque, Exposé XI (1987), 221-243.

[C] Chern, S.S. On special $W$-surfaces. Proc. A.M.S., (1955), 783-786.

[CL] Coddington, E.A. \& Levinson, N. Theory of ordinary differential equations. McGraw-Hill, New York, 1955.

[F] Furuya, S. On periods of periodic solutions of a certain non-linear differential equation. Japan - U.S. Seminar on Ord. Diff. and Functional Eq., Springer-Verlag, Berlin and New York (1971), 320-323.

[HW] Hartman, P. \& Wintner, A. Umbilical points and $W$-surfaces. Amer. J. of Math.. 76 (1954), 502-508. 
[Ho] Hopf, H. Differential geometry in the large. Lect. Notes in Math.. Springer-Verlag, 1000, 1983.

[H] Hsiang, W.Y. On rotational $W$-hypersurfaces in spaces of constant curvature and generalized laws of sine and cosine. Bull. Inst. Acad. Sinica, 11 (1983), 349-373.

[HL] Hsiang, W.Y. \& Lawson Jr.. B.L. Minimal submanifolds of low cohomogeneity. J. Diff. Geom., 5 (1971), 1-38.

[L] Lawson, B. Complete minimal surfaces in $S^{3}$. Ann. of Math., 90 (1970), 335-374.

[M] Mori, H. The first eingenvalue of Laplacians on minimal surfaces in $S^{3}$. J. Math. Soc. Japan, 37 (1985), 79-86.

[O1] Otsuki, T. Minimal hypersurfaces in a Riemannian manifold of constant curvature. Amer. J. Math., 92 (1970), 145-173.

[O2] Otsuki, T. On integral inequalities related with a certain non-linear differential equation. Proc. Japan Acad., 48 (1972), 9-12.

[R1] Ripoll, J. Superfícies invariantes de curvatura média constante. Tese de Doutorado, IMPA, Rio de Janeiro, 1986.

[R2] Ripoll, J. Uniqueness of minimal rotational surfaces in $S^{3}$. Amer. J. of Math., 111 (1989), 537-547.

[SaET1] Sa Earp, R. \& Toubiana, E. A note on special surfaces in $\mathbb{R}^{3}$. Matemática Contemporânea, 4 (1993), 109-118.

[SaET2] Sa Earp, R. \& Toubiana, E. Sur les surfaces spéciales de type minimal. Preprint. 